\title{
Universidade de São Paulo Instituto de Ciências Matemáticas de São Carlos
}

\author{
Algoritmos de Pontos Interiores para \\ Programação Linear e uma Extensão \\ para a Programação Linear por Partes
}

Luiza Takako Matumoto

Orientador: Prof. Dr. Marcos Nereu Arenales

Dissertação apresentada ao Instituto de Ciências Matemáticas de São Carlos da Universidade de São Paulo - ICMSC/USP, como parte dos requisitos para a obtenção do título de Mestre em Ciências - Área: Ciências de Computação e Matemática Computacional.

São Carlos

1996 
À minha far

pela compreensão, apoio e ince 
das coisas

que eu fiz a metro

todos saberão

quantos quilômetros

são

aquelas

em centímetros

sentimentos mínimos

impetos infinitos

não?

( Paulo Leminski ) 


\section{Agradecimentos}

Ao Prof. Dr. Marcos Nereu Arenales, pela orientação, acompanhamento, paciência e sobretudo amizade.

À minha família e à minha mãe em especial, que souberam compreender minha ausência e que sempre dão incentivo para o meu crescimento emocional e profissional.

Às secretárias da Pós-Graduação Beth, Laura e Marília, pela presteza e por sempre estarem dispostas a ajudar no que fosse preciso.

Pela amizade, conforto e por tornar mais terna a minha vida agradeço de coração aos vários amigos que fiz durante o Mestrado, e que pela preocupação de não esquecer de ninguém, omitirei a maioria dos nomes, citando apenas como sendo o pessoal do Coral Boca em Cena, a turma dos jogos de baralho, as garotas das repúblicas onde morei e vizinhos, bem como outros amigos feitos durante a permanência na cidade e no decorrer do programa de Mestrado.

Ao Márcio pela ajuda na digitação parcial deste trabalho e mais pelo apoio, carinho e amizade que me dedicou.

À Simone, Rosana e Cidinho pela cumplicidade nos momentos difíceis.

À Ana Cristina e Luís Maurício pela colaboração para este trabalho.

Aos funcionários que de uma maneira ou de outra contribuíram para a realização deste trabalho.

À Capes, pelo apoio financeiro.

A Deus, por me acompanhar em todos os momentos. 
Desde o trabalho de Karmarkar em 1984, intensas pesquisas têm sido feitas sobre métodos de pontos interiores. Nesta dissertação estudamos a bibliografia relacionada a métodos de pontos interiores para a programação linear com ênfase em alguns algoritmos básicos: métodos de escala-afim primal e dual e método (path-following) primal-dual. Por último mostramos como estender algoritmos primais-duais a uma classe de programação linear por partes.

\section{Abstract}

Since Karmarkar's work in 1984, intensive research has been carried out on interior point methods. In this dissertation we studied the related bibliography of interior point methods for linear programming focusing on some basic algorithms: primal and dual affine-scaling methods and primal-dual (path-following) method. Finally it was showed how to extend primal-dual algorithms to a class of piecewise linear programming. 


\section{Índice}

Capítulo 1 - Introdução.

Capítulo 2 - Preliminares...............................................................................................04

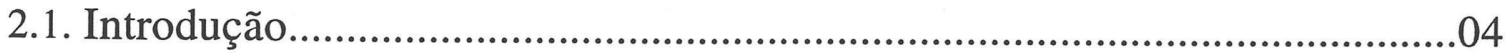

2.2. Alguns conceitos básicos da programação linear...........................................04

2.3. Alguns conceitos básicos da programação-não-linear........................................11

Capítulo 3 - Métodos de Escala-Afim Primal e Dual..............................................17

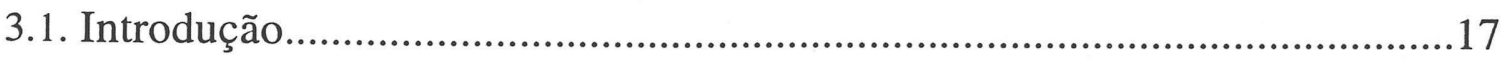

3.2. Método de Escala-Afim Primal..............................................................18

3.2.1. Mudança de escala.....................................................................18

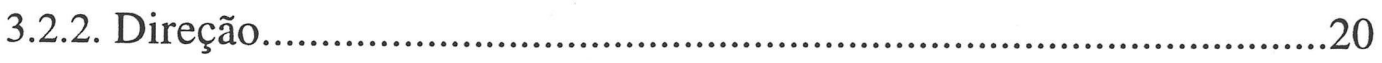

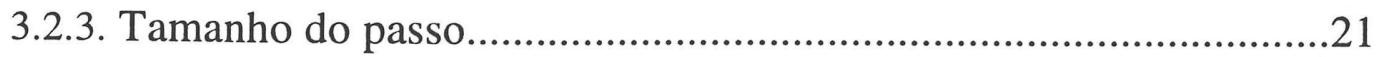

3.2.4. Algoritmo de Escala-Afim Primal....................................................22

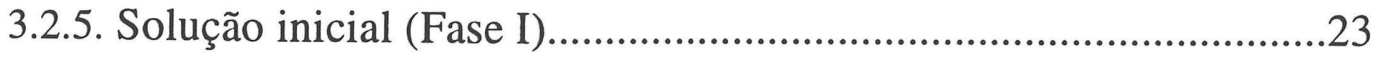

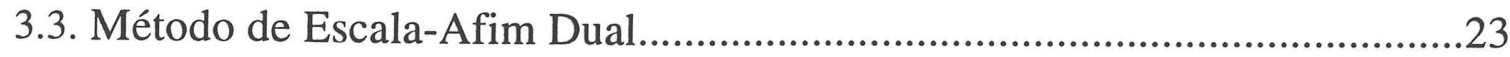

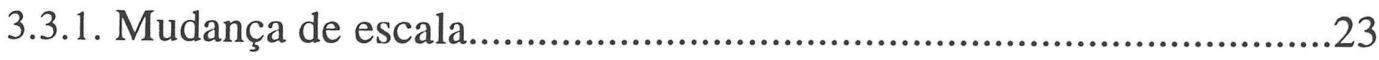

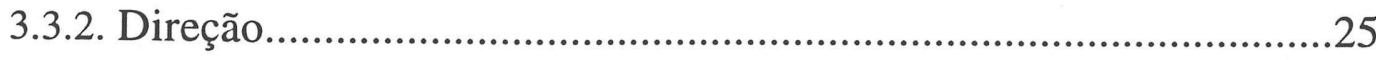

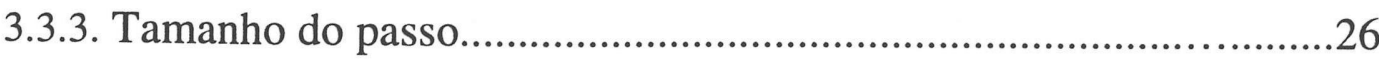

3.3.4. Algoritmo de Escala-Afim Dual.......................................................27

3.3.5. Solução inicial (Fase I)........................................................................28

Capítulo 4 - Método Primal-Dual.....................................................................................30

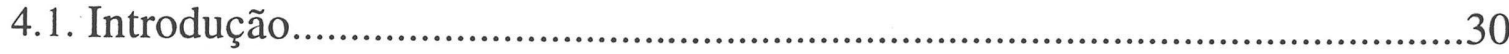

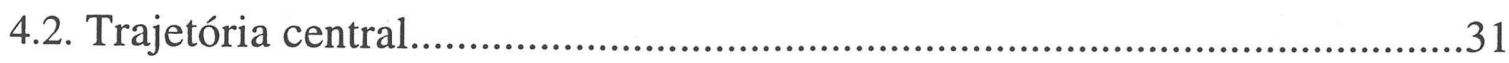

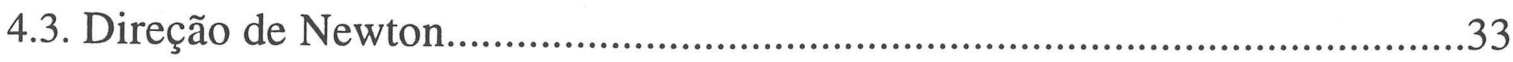

4.4. Tamanho do passo e o parâmetro de penalização............................................36 


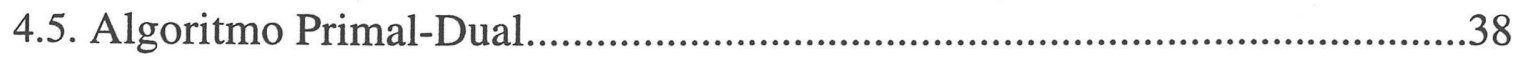

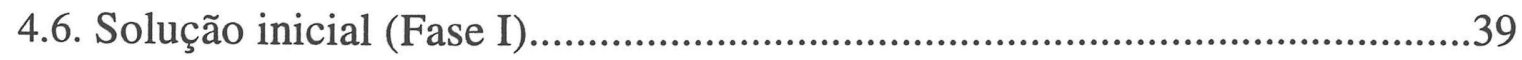

Capítulo 5 - Extensão do método primal-dual........................................................41

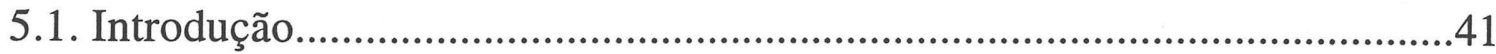

5.2. Definição de um problema linear por partes...................................................41

5.3. O problema dual e condições de otimalidade...............................................41

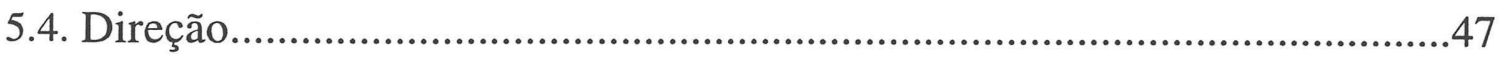

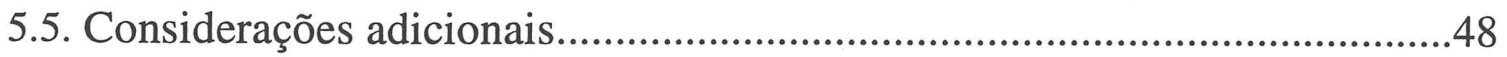

Capítulo 6 - Conclusões e Considerações finais.........................................................51

Referências Bibliográficas....................................................................................54 


\section{Capítulo 1}

\section{INTRODUÇÃO}

A história da programação linear teve um marco fundamental em 1947 quando G. B. Dantzig desenvolveu o método simplex para solucionar problemas de programação linear. O que se seguiu foi um rápido desenvolvimento neste campo.

O método simplex mostrou-se excelente em aplicações práticas, embora o desempenho teórico seja insatisfatório devido a complexidade exponencial.. Um exemplo que ilustra a complexidade exponencial foi apresentado por V. Klee e G. J. Minty em 1972 (ver [CHV83]). O método simplex para solucionar o problema

$$
\begin{aligned}
&\left(\mathbf{P}_{\text {ex }}\right) \quad \text { Maximize } \sum_{\mathrm{j}=1}^{\mathrm{n}} 10^{\mathrm{n}-\mathrm{j}} \mathrm{x}_{\mathrm{j}} \\
& \text { sujeito à }\{\left\{\left(2 \sum_{\mathrm{j}=1}^{\mathrm{n}} 10^{\mathrm{i}-\mathrm{j}} \mathrm{x}_{\mathrm{j}}\right)+\mathrm{x}_{\mathrm{i}} \leq 10^{\mathrm{i}-1} \quad(\mathrm{i}=1, \ldots, \mathrm{n})\right. \\
& \mathrm{x}_{\mathrm{j}} \geq 0 \quad(\mathrm{j}=1, \ldots, \mathrm{n})
\end{aligned}
$$

necessita de $2^{\mathrm{n}}-1$ iterações, o que mostra ser o algoritmo não-polinomial.

Em 1979 surgiu o "método dos elipsóides" de classe polinomial apresentado por L. G. Khachiyan (ver [GON89]) que obteve uma complexidade de $\mathrm{O}\left(\mathrm{n}^{4} \mathrm{~L}\right)$ operações aritméticas (ver [MAR91]). Tal método, entretanto, mostrou-se ineficiente em problemas práticos, embora tivesse propriedades teóricas boas.

Em 1984 N. Karmarkar publicou um algoritmo projetivo para a programação linear [KAR84], o primeiro método primal de redução de potencial. Com este trabalho, Karmarkar 
conseguiu uma redução de complexidade polinomial em relação ao "método dos elipsóides". Karmarkar obteve um limite para o número de iterações de $\mathrm{O}(\mathrm{nL})$, e um limite para o número de operações aritméticas de $\mathrm{O}\left(\mathrm{n}^{3.5} \mathrm{~L}\right)$ [GON90]. Outro aspecto relevante é que o método de Karmarkar comparado ao "método dos elipsóides" era bem mais eficiente do ponto de vista prático.

O novo algoritmo não somente superou o método simplex na teoria, como também mostrou um enorme potencial para solucionar problemas práticos de grande porte. Trabalha também radicalmente diferente do método simplex, pois o algoritmo de Karmarkar procura a solução ótima pelo interior da região de factibilidade. Este trabalho levou a intensas pesquisas de métodos de pontos interiores para a programação linear e o algoritmo no formato original, por ser muito complexo, vem sendo alterado a partir de 1985 por vários pesquisadores com melhoramentos ao método e desenvolvimento de variantes.

Uma simplificação do algoritmo projetivo de Karmarkar gerou o método escala-afim. Este método utiliza uma transformação afim em detrimento à transformação projetiva original de Karmarkar e muitos pesquisadores optaram por trabalhar os problemas de programação linear no formato padrão.

Entretanto, o algoritmo de escala-afim básico foi primeiramente apresentado por I. I. Dikin em 1967. Posteriormente, em 1985, foi redescoberto simultaneamente por E. Barnes e R. Vanderbei, M. Meketon, e B. Freedman, os quais propuseram o algoritmo de escala-afim primal para solucionar o problema linear na forma padrão e estabeleceram a prova da convergência do algoritmo (ver [FAN93]).

Um algoritmo semelhante chamado algoritmo de escala-afim dual foi desenvolvido e implementado por I. Adler, N. Karmarkar, M. G. C. Resende e G. Veiga para solucionar problemas lineares na forma de desigualdade [ADL89].

Uma outra variante que incorpora a função barreira logarítmica aos algoritmos escalaafim primal e dual é o chamado algoritmo primal-dual. Foi apresentado e analisado por R. Monteiro, I. Adler, e M. G. C. Resende e por M. Kojima, S. Mizuno, e A. Yoshise em 1987 (ver [FAN93]), entre outros. Vários estudos foram realizados baseados nos algoritmos primais-duais nos últimos anos.

No Capítulo 2 fazemos uma breve revisão de conceitos e resultados que serão utilizados nos demais capítulos.

O Capítulo 3 é reservado aos algoritmos primal e dual, que são as variantes mais simples do algoritmo original de Karmarkar.

O Capítulo 4 é dedicado ao algoritmo primal-dual, também chamado de algoritmo escala-afim primal-dual [FAN93] ou algoritmo path-following primal-dual [FOU91][VAN94][etc]. 
No Capítulo 5 temos uma extensão do método primal-dual estudado no Capítulo 4, para tratar um problema linear por partes.

Finalmente, no Capítulo 6, fazemos algumas considerações adicionais sobre os métodos estudados e perspectivas em face da literatura lida. 


\section{PRELIMINARES}

\subsection{Introdução}

Neste capítulo revemos alguns conceitos básicos da programação linear e não-linear [BAZ77][CHV83][LUE84], que serão utilizados posteriormente, para a apresentação e desenvolvimento de métodos de pontos interiores para programação linear. Estes conceitos estão dispostos em duas seções, uma referente à programação linear (PL) e outra referente à programação não-linear (PNL).

\subsection{Alguns conceitos básicos da programação linear}

$$
\text { Sejam } c=\left(c_{1}, \ldots, c_{n}\right)^{t}, b=\left(b_{1}, \ldots, b_{m}\right)^{t}, x=\left(x_{1}, \ldots, x_{n}\right)^{t} \text { e } A=\operatorname{matriz}\left(a_{i j}\right) . \quad \text { Um }
$$
problema de programação linear (formato padrão) pode ser escrito como se segue:

$$
\begin{aligned}
& \text { (P) Minimize } \mathbf{c}^{\mathrm{t}} \mathbf{x} \\
& \text { sujeito à }\left\{\begin{array}{c}
\mathbf{A x}=\mathbf{b} \\
\mathbf{x} \geq \mathbf{0}
\end{array}\right.
\end{aligned}
$$

Assumimos, sem perda de generalidades, que a matriz de restrições A tem posto máximo. O problema $(\mathrm{P})$ será referido como problema primal.

$\mathrm{O}$ conjunto factível do problema $(\mathrm{P})$ é dado por: 


$$
S=\left\{x \in \Re^{n} \mid A x=b ; x \geq 0\right\}
$$

Chamamos de Interior Relativo de $\mathrm{S}$ ao conjunto:

$$
\stackrel{\circ}{S}=\left\{x \in \Re^{n} \mid A x=b ; x>0\right\}
$$

como sendo o conjunto das soluções factíveis interiores de $(\mathrm{P})$. Suporemos que $\stackrel{\dot{S}}{\mathrm{~S}} \neq \varnothing$.

Denotaremos uma matriz diagonal $\mathrm{n} \times \mathrm{n}$, em relação a $\mathrm{x}$, por:

$$
X=\operatorname{diag}\left(\mathrm{x}_{1}, \ldots, \mathrm{x}_{\mathrm{n}}\right)=\left[\begin{array}{cccc}
\mathrm{x}_{1} & 0 & \cdots & 0 \\
0 & \mathrm{x}_{2} & \cdots & 0 \\
\vdots & \vdots & \ddots & \vdots \\
0 & 0 & \cdots & \mathrm{x}_{\mathrm{n}}
\end{array}\right]
$$

Observemos que a matriz $X$ é não singular quando $x \in \stackrel{\circ}{S}$, com uma inversa $X^{-1}$ dada por:

$$
\mathrm{X}^{-1}=\operatorname{diag}\left(\frac{1}{\mathrm{x}_{1}}, \ldots, \frac{1}{\mathrm{x}_{\mathrm{n}}}\right)=\left[\begin{array}{cccc}
1 / \mathrm{x}_{1} & 0 & \cdots & 0 \\
0 & 1 / \mathrm{x}_{2} & \cdots & 0 \\
\vdots & \vdots & \ddots & \vdots \\
0 & 0 & \cdots & 1 / \mathrm{x}_{\mathrm{n}}
\end{array}\right]
$$

Esta notação será bastante utilizada nos demais capítulos, inclusive em relação às outras variáveis.

Um conceito importante em PL, que será utilizado nos demais capítulos, é a noção de dualidade.

Considere o problema $(\mathrm{P})$. Associamos à restrição $\mathrm{Ax}=\mathrm{b}$ uma variável $\mathrm{y}$. A função lagrangeana para $\mathrm{x}$ e y é definida por:

$$
L(x, y)=c^{t} x+y^{t}(b-A x)=\left(c^{t}-y^{t} A\right) x+y^{t} b
$$

O problema lagrangeano (ou função dual) é dado por:

$$
l(y)=\min _{x \geq 0} L(x, y)
$$


Segue que:

$$
1(y) \leq c^{t} x \text {, para todo } x \text { factível, }
$$

ou seja, a função dual fornece um limitante inferior para a função objetivo primal. A escolha de y, que fornece o melhor limitante inferior, define o problema dual:

$$
\begin{aligned}
& \text { (D) } \\
& y \in \mathfrak{R}^{\mathrm{m}}
\end{aligned}
$$

A seguir expressamos a solução do problema lagrangeano.

$$
l(y)=\min _{x \geq 0} L(x, y)=\min _{x \geq 0}\left(c^{t}-y^{t} A\right) x+y^{t} b
$$

Primeiramente, façamos uma representação componente a componente do problema.

$$
\left(c^{t}-y^{t} A\right) x=\sum_{i=1}^{n}\left(c_{i}-y^{t} a_{i}\right) x_{i}
$$

onde $\mathrm{a}_{\mathrm{i}}$ são as colunas da matriz $\mathrm{A}$.

$$
\begin{aligned}
\min _{x \geq 0} L(x, y) & =\min _{x \geq 0} \sum_{i=1}^{n}\left(c_{i}-y^{t} a_{i}\right) x_{i}+y^{t} b \\
& =\sum_{i=1}^{n} \min _{x_{i} \geq 0}\left(c_{i}-y^{t} a_{i}\right) x_{i}+y^{t} b
\end{aligned}
$$

Façamos uma análise do problema:

Se $c_{i}-y^{t} a_{i}<0$, sendo $x_{i} \geq 0$, então $l(y)$ não é limitante para o problema $(1(y) \rightarrow-\infty)$. Logo,

$$
c_{i}-y^{t} a_{i} \geq 0, i=1, \ldots, n
$$

ou seja,

$$
c^{t}-y^{t} A \geq 0 \Rightarrow A^{t} y \leq c
$$


Se y é tal que $\mathrm{A}^{\mathrm{t}} \mathrm{y} \leq \mathrm{c}$, então:

$$
\min _{x_{i} \geq 0}\left(c_{i}-y^{t} a_{i}\right) x_{i}=0
$$

e, portanto, a função dual encontrada é:

$$
l(y)=b^{t} y \text { se } A^{t} y \leq c
$$

Depois dessas considerações, o problema dual se escreve por:

\section{(D) Maximize $\mathbf{b}^{\mathbf{t}} \mathbf{y}$ sujeito à $\left\{\begin{array}{c}\mathbf{A}^{t} \mathbf{y} \leq \mathbf{c} \\ y \text { irrestrito }\end{array}\right.$}

O conjunto factível do problema (D) é dado por:

$$
\mathrm{T}=\left\{(\mathrm{y}, \mathrm{z}) \in \mathfrak{R}^{\mathrm{m}} \times \Re^{\mathrm{n}} \mid \mathrm{A}^{\mathrm{t}} \mathrm{y}+\mathrm{z}=\mathrm{c}, \mathrm{z} \geq 0\right\}
$$

Chamamos de Interior Relativo de T ao conjunto:

$$
\stackrel{\circ}{\mathrm{T}}=\left\{(\mathrm{y}, \mathrm{z}) \in \mathfrak{R}^{\mathrm{m}} \times \mathfrak{R}^{\mathrm{n}} \mid \mathrm{A}^{\mathrm{t}} \mathrm{y}+\mathrm{z}=\mathrm{c} ; \mathrm{z}>0\right\}
$$

como sendo o conjunto das soluções factíveis interiores de (D). Suporemos $\stackrel{\circ}{\mathrm{T}} \neq \varnothing$.

Denotaremos, analogamente a (2.2.3) e (2.2.4), a matriz diagonal associada a z por:

$$
\mathrm{Z}=\operatorname{diag}\left(\mathrm{z}_{1}, \ldots, \mathrm{z}_{\mathrm{n}}\right)=\left[\begin{array}{cccc}
\mathrm{z}_{1} & 0 & \cdots & 0 \\
0 & \mathrm{z}_{2} & \cdots & 0 \\
\vdots & \vdots & \ddots & \vdots \\
0 & 0 & \cdots & \mathrm{z}_{\mathrm{n}}
\end{array}\right]
$$

Se $\mathrm{z} \in \stackrel{\circ}{\mathrm{T}}$, então $\mathrm{Z}$ é não singular, e sua inversa é dada por: 


$$
\mathrm{Z}^{-1}=\operatorname{diag}\left(\frac{1}{\mathrm{z}_{1}}, \ldots, \frac{1}{\mathrm{z}_{\mathrm{n}}}\right)=\left[\begin{array}{cccc}
1 / \mathrm{z}_{1} & 0 & \cdots & 0 \\
0 & 1 / \mathrm{z}_{2} & \cdots & 0 \\
\vdots & \vdots & \ddots & \vdots \\
0 & 0 & \cdots & 1 / \mathrm{z}_{\mathrm{n}}
\end{array}\right]
$$

Adotando um procedimento análogo ao utilizado para encontrar o problema dual de um problema linear $(\mathrm{P})$, acharemos o problema dual de um problema linear com variáveis canalizadas $\left(\mathrm{P}_{\mathrm{VCan}}\right)$.

Considere agora o seguinte problema com variável canalizada:

$$
\begin{array}{ll}
\left(\mathbf{P}_{\text {VCan }}\right) & \text { Minimize } \mathbf{c}^{\mathbf{t}} \mathbf{x} \\
& \text { sujeito à }\left\{\begin{array}{c}
A \mathbf{A}=\mathbf{b} \\
\mathbf{u}^{-} \leq \mathrm{x} \leq \mathbf{u}^{+}
\end{array}\right.
\end{array}
$$

onde $\mathrm{u}^{-}<\mathrm{u}^{+}$.

Encontraremos o problema dual associado ao problema $\left(\mathrm{P}_{\mathrm{VCan}}\right)$. Para tanto, construímos a função langrangeana e resolvemos o problema lagrangeano (função dual).

Associamos à restrição $\mathrm{Ax}=\mathrm{b}$, do problema $\left(\mathrm{P}_{\mathrm{VCan}}\right)$, uma variável $\mathrm{y}$. A função lagrangeana para $\mathrm{x}$ e y é dada por:

$$
L(x, y)=c^{t} x+y^{t}(b-A x)=\left(c^{t}-y^{t} A\right) x+y^{t} b, \quad \text { para } u^{-} \leq x \leq u^{+}
$$

O problema lagrangeano (ou função dual) é dado por:

$$
1(y)=\min _{u^{-} \leq x \leq u^{+}} L(x, y)
$$

Decorre da definição de l(y) que:

$$
l(y) \leq c^{t} x, \quad \text { para todo } x \text { factível }
$$

Portanto, vemos que a função dual fornece um limitante inferior para a função objetivo primal e a escolha de y, que fornece o melhor limitante inferior, define o problema dual, ou seja,

$$
\begin{aligned}
& \left(\mathrm{P}_{\mathrm{VCan}}\right) \quad \text { Maximize } \mathrm{l}(\mathrm{y}) \\
& y \in \mathfrak{R}^{\mathrm{m}}
\end{aligned}
$$


A resolução da função dual é dado a seguir.

O desenvolvimento de obtenção de (2.2.9) é válido:

$$
1(y)=\min _{u^{-} \leq x \leq u^{+}} L(x, y)=\sum_{i=1}^{n} \min _{u_{i}^{-} \leq x_{i} \leq u_{i}^{+}}\left(c_{i}-y^{t} a_{i}\right) x_{i}+y^{t} b
$$

onda $\mathrm{a}_{\mathrm{i}}$ são colunas da matriz A.

Analisando o problema vemos que $\left(c_{i}-y^{t} a_{i}\right)$ pode ser estritamente positivo, estritamente negativo, ou nulo.

Definamos, para simplicidade de notação,

$$
z^{t}=c^{t}-y^{t} A
$$

Se $z_{i}>0$ então $\min _{u_{i}^{-} \leq x_{i} \leq u_{i}^{+}} z_{i} x_{i}=z_{i} u_{i}^{-}$

Se $\mathrm{z}_{\mathrm{i}}=0$ então $\min _{\mathrm{u}_{\mathrm{i}}^{-} \leq \mathrm{x}_{\mathrm{i}} \leq \mathrm{u}_{\mathrm{i}}^{+}} \mathrm{z}_{\mathrm{i}} \mathrm{x}_{\mathrm{i}}=0$ para $\forall \mathrm{x}_{\mathrm{i}} \in\left[\mathrm{u}_{\mathrm{i}}^{-}, \mathrm{u}_{\mathrm{i}}^{+}\right]$

Se $z_{i}<0$ então $\min _{u_{i}^{-} \leq x_{i} \leq u_{i}^{+}} z_{i} x_{i}=z_{i} u_{i}^{+}$

Considerando (2.2.21)-(2.2.23), temos:

Maximize $\mathrm{l}(\mathrm{y})=\mathrm{y}^{\mathrm{t}} \mathrm{b}+\mathrm{u}^{\mp} \mathrm{z}$
sujeito à $\left\{\begin{array}{c}\mathrm{z}^{\mathrm{t}}=\mathrm{c}^{\mathrm{t}}-\mathrm{y}^{\mathrm{t}} \mathrm{A} \\ \mathrm{y} \in \Re^{\mathrm{m}}\end{array}\right.$

onde $\mathrm{u}^{\mp} \mathrm{z}=\sum_{\mathrm{i}=1}^{\mathrm{n}} \mathrm{u}_{\mathrm{i}}^{\mp} \mathrm{z}_{\mathrm{i}}$ é uma soma de funções lineares por partes côncavas com:

$$
u_{i}^{\mp} z_{i}= \begin{cases}u_{i}^{-} z_{i} & \text { se } z_{i} \geq 0 \\ u_{i}^{+} z_{i} & \text { se } z_{i} \leq 0\end{cases}
$$

ou seja,

\begin{tabular}{|ll|}
\hline$\left(D_{\text {VCan }}\right)$ & Maximize $\mathbf{b}^{t} \mathbf{y}+\mathbf{u}^{\mp} \mathbf{z}$ \\
& sujeito à $\left\{\begin{array}{c}\mathbf{A}^{\mathrm{t}} \mathbf{y}+\mathbf{z}=\mathbf{c} \\
\mathbf{y} \in \Re^{\mathrm{m}}\end{array}\right.$ \\
\hline
\end{tabular}

No capítulo 5, o problema dual para um problema linear por partes será apresentado. 
Um importante resultado da PL que relaciona os problemas (P) e (D) é enunciado a seguir:

Teorema 2.2.1: (Condições de Otimalidade de Karush-Kuhn-Tucker (KKT) para PL) Considere o problema (P). O vetor x é uma solução ótima do problema (P) e (y,z) é solução ótima de (D) se, e somente se,

$$
\begin{array}{ll}
\mathbf{A x}=\mathbf{b}, \mathbf{x} \geq \mathbf{0} & \text { (factibilidade primal) } \\
\mathbf{A}^{t} \mathbf{y}+\mathbf{z}=\mathbf{c}, \mathbf{z} \geq \mathbf{0} & \text { (factibilidade dual) } \\
\mathbf{z}^{t} \mathbf{x}=\mathbf{0} & \text { (folgas complementares) }
\end{array}
$$

As condições (KKT) para PNL serão enunciadas na próxima seção.

Uma outra relação entre $(\mathrm{P})$ e (D) diz respeito à solução ótima.

Qualquer que seja x primal factível e qualquer que seja y dual factível temos:

$$
b^{t} y \leq c^{t} x
$$

onde $c^{t} x$ e $b^{t} y$ são as funções objetivos dos problemas (P) e (D), respectivamente.

Se a solução ótima $x^{*}$ existe então a solução ótima $y^{*}$ existe e $b^{t} y^{*}=c^{t} x^{*}$. Logo temos que o valor ótimo satisfaz:

$$
b^{t} y \leq \text { valor ótimo } \leq c^{t} x
$$

Considere o problema (P). Uma direção d é factível se pertencer ao espaço nulo da matriz de restrições $\mathrm{A}$, ou seja,

$$
\operatorname{Ad}=0
$$

Isto se deve ao fato de que, para que $\mathrm{d}$ seja direção factível, então $\mathrm{x}+\alpha \mathrm{d}$, para $\alpha>0$, pequeno, deve também ser factível. Logo temos,

$$
\mathrm{A}(\mathrm{x}+\alpha \mathrm{d})=\mathrm{b}, \text { que implica em, } \alpha \mathrm{Ad}=0
$$

Portanto, $\mathrm{Ad}=0$.

Além disso, se $x_{i}=0$, como $x+\alpha d \geq 0$, segue que $d_{i} \geq 0$. Em particular, se $x$ é interior, então $\mathrm{Ad}=0$ é condição necessária e suficiente para que d seja direção factível em $\mathrm{x}$. 
Uma estratégia usada para determinar direções de busca é a de projetar ortogonalmente o gradiente sobre o núcleo de A (este corresponde ao elemento mais próximo do gradiente). Esta estratégia é usada por muitos métodos de otimização.

Definimos a matriz de projeção no espaço nulo por

$$
P=I-A^{t}\left(A A^{t}\right)^{-1} A
$$

Um procedimento para obtenção de (2.2.28) é encontrado em [GON89].

No caso de problemas de minimização, em especial do problema $(\mathrm{P})$, a direção de descida d que será usada posteriormente, será a do vetor oposto ao vetor gradiente da função objetivo, projetada no espaço nulo da matriz de restrições, isto é,

$$
d=P(-c)
$$

\subsection{Alguns conceitos básicos da programação não-linear}

O teorema 2.2.1, da seção anterior, é um importante resultado para a PL, e é um caso especial do resultado geral das condições KKT para a PNL, que será enunciada nesta seção.

Primeiramente, consideremos um problema da seguinte forma:

\begin{tabular}{|l|l|}
\hline (PN) Minimize & $\mathbf{f}(\mathbf{x})$ \\
sujeito à & $\left\{\begin{array}{cc}\mathbf{p}_{1}(\mathbf{x})=\mathbf{0} & \mathbf{q}_{1}(\mathbf{x}) \leq \mathbf{0} \\
\mathbf{p}_{2}(\mathbf{x})=\mathbf{0} & \mathbf{q}_{2}(\mathbf{x}) \leq \mathbf{0} \\
\vdots & \vdots \\
\mathbf{p}_{\mathrm{m}}(\mathbf{x})=\mathbf{0} & \mathbf{q}_{\mathrm{r}}(\mathbf{x}) \leq \mathbf{0} \\
\mathbf{x} \in \Omega \subset \mathfrak{R}^{\mathbf{n}}\end{array}\right.$ \\
\hline
\end{tabular}

onde $m \leq n$ e as funções $f, p_{i}, i=1, \ldots, m$ e $q_{j}, j=1, \ldots, r$ são de classe $C^{1}$.

Para simplificar a notação, façamos $p=\left(p_{1}, p_{2}, \ldots, p_{m}\right)$ e $q=\left(q_{1}, q_{2}, \ldots, q_{r}\right)$ e reescrevemos (PN) como: 


\section{(PN) Minimize $\mathbf{f}(\mathbf{x})$ \\ sujeito à $\left\{\begin{array}{c}p(x)=0 \\ q(x) \leq 0 \\ x \in \Omega\end{array}\right.$}

Definicão 2.3.1: Seja x um ponto que satisfaz as restrições,

$$
\mathrm{p}(\mathrm{x})=0, \mathrm{q}(\mathrm{x}) \leq 0
$$

e seja $\mathrm{J}$ o conjunto de índices $\mathrm{j}$ para cada $\mathrm{q}_{\mathrm{j}}(\mathrm{x})=0$. Então $\mathrm{x}$ é chamado de ponto regular das restrições (2.3.1) se os vetores gradiente $\nabla \mathrm{p}_{\mathrm{i}}(\mathrm{x}), \nabla \mathrm{q}_{\mathrm{j}}(\mathrm{x}), 1 \leq \mathrm{i} \leq \mathrm{m}, \mathrm{j} \in \mathrm{J}$ são linearmente independentes.

Teorema 2.3.1: (Condição de Otimalidade de $1^{\circ}$ Ordem ou Condição (KKT) para PNL) Considere o problema (PN). Seja x um ponto de mínimo local de $\mathrm{f}(\mathrm{x})$ restrito a (2.3.1) e regular. Então existem vetores $u$ e $v, v \geq 0$, tais que,

$$
\begin{aligned}
& \nabla \mathrm{f}(\mathrm{x})+\mathrm{u}^{\mathrm{t}} \nabla \mathrm{p}(\mathrm{x})+\mathrm{v}^{\mathrm{t}} \nabla \mathrm{q}(\mathrm{x})=0 \\
& \mathrm{v}^{\mathrm{t}} \mathrm{q}(\mathrm{x})=0
\end{aligned}
$$

onde $\nabla \mathrm{p}$ e $\nabla \mathrm{q}$ são as matrizes jacobianas de p e $\mathrm{q}$.

Teorema 2.3.2: (Condição de Otimalidade de $2^{\circ}$ Ordem) Considere o problema (PN) supondo funções $\mathrm{f}$, $\mathrm{p}$ e $\mathrm{q} \in \mathrm{C}^{2}$ e $\mathrm{x}$ um ponto regular das restrições (2.3.1). Se $\mathrm{x}$ é um ponto de mínimo local, então existem vetores u e v, v $\geq 0$, tais que (2.3.2) e (2.3.3) são satisfeitos e,

$$
\mathrm{H}(\mathrm{x})=\nabla^{2} \mathrm{f}(\mathrm{x})+\mathrm{u}^{\mathrm{t}} \nabla^{2} \mathrm{p}(\mathrm{x})+\mathrm{v}^{\mathrm{t}} \nabla^{2} \mathrm{q}(\mathrm{x})
$$

é definida semi-positiva sobre o subespaço tangente das restrições ativas em $\mathrm{x}$, onde $\mathrm{u}^{\mathrm{t}} \nabla^{2} \mathrm{p}(\mathrm{x})=\sum_{\mathrm{i}=1}^{\mathrm{m}} \mathrm{u}_{\mathrm{i}} \nabla^{2} \mathrm{p}_{\mathrm{i}}(\mathrm{x})$ e $\nabla^{2} \mathrm{p}_{\mathrm{i}}(\mathrm{x})$ é a matriz hessiana de $\mathrm{p}$.

Se o problema (PN) for convexo, isto é, se a função objetivo $f(x)$ for convexa e as funções-restrições $\mathrm{p}_{\mathrm{i}}(\mathrm{x})$ são lineares e $\mathrm{q}_{\mathrm{j}}(\mathrm{x})$ convexas, então as condições (KKT) são também suficientes. 
Teorema 2.3.3: (Condição suficiente para problemas convexos) Suponha que o problema (PN) seja convexo. Se x é ponto factível, regular e satisfaz as condições (2.3.2) e (2.3.3) então x é solução ótima de $(\mathrm{PN})$.

Este teorema será empregado no desenvolvimento de métodos do tipo primal-dual quando aplicado ao seguinte problema-barreira:

$$
\begin{array}{r}
\left(\mathbf{P}_{\mu}\right) \quad \text { Minimize } \mathbf{c}^{\mathrm{t}} \mathbf{x}-\mu \sum_{\mathrm{j}=1}^{\mathrm{n}} \ln \left(\mathrm{x}_{\mathrm{i}}\right) \\
\text { sujeito à }\left\{\begin{array}{c}
\mathrm{Ax}=\mathrm{b} \\
\mathrm{x}>0
\end{array}\right.
\end{array}
$$

A solução do problema $\left(\mathrm{P}_{\mu}\right)$ para cada $\mu>0$ é único e decorre do fato de o problema $\left(\mathrm{P}_{\mu}\right)$ ser estritamente convexo. Este mínimo está completamente caracterizado pelas condições de Karush-Kuhn-Tucker(condições KKT), expresso no Teorema 2.3.1:

$$
\begin{aligned}
& c-\mu X^{-1} e-A^{t} y=0 \\
& A x=b, x>0
\end{aligned}
$$

onde $e=(1,1, \ldots, 1)^{t}$ e $X=\operatorname{diag}\left(x_{1}, \ldots, x_{n}\right)$.

Introduzindo o vetor $\mathrm{z}=\mu \mathrm{X}^{-1} \mathrm{e}$, o sistema acima pode ser reescrito da seguinte forma:

$$
\begin{array}{ll}
\mathrm{Ax}=\mathrm{b}, \mathrm{x}>0 & \text { (factibilidade primal) } \\
\mathrm{A}^{\mathrm{t}} \mathrm{y}+\mathrm{z}=\mathrm{c} & \text { (factibilidade dual) } \\
\mathrm{XZe}-\mu \mathrm{e}=0 & \text { (folgas complementares) }
\end{array}
$$

onde $\mathrm{Z}=\operatorname{diag}\left(\mathrm{z}_{1}, \ldots, \mathrm{z}_{\mathrm{n}}\right)$ e $\mathrm{z}=\mathrm{Ze}$.

Note que a equação (2.3.6c) pode ser escrita componente a componente como:

$$
\mathrm{x}_{\mathrm{j}} \mathrm{z}_{\mathrm{j}}=\mu \quad \text { para } \mathrm{j}=1, \ldots, \mathrm{n}
$$

o que implica que $\mathrm{z}>0$ e portanto, $(\mathrm{y}, \mathrm{z}) \in \stackrel{\circ}{\mathrm{T}}$.

O sistema (2.3.6), além de caracterizar a solução do problema $\left(\mathrm{P}_{\mu}\right)$, também fornece a solução para o seguinte problema: 


$$
\begin{aligned}
& \left(D_{\mu}\right) \text { Maximize } b^{t} y+\mu \sum_{j=1}^{n} \ln \left(z_{j}\right) \\
& \text { sujeito à }\left\{\begin{array}{l}
A^{t} y+z=c \\
z>0
\end{array}, y\right. \text { irrestrito }
\end{aligned}
$$

O problema $\left(D_{\mu}\right)$ é a versão penalizada do problema dual (D), analogamente a $\left(P_{\mu}\right)$.

Para verificar que a solução de (2.3.6) fornece também a solução de $\left(D_{\mu}\right)$, para cada $\mu>0$ fixo, considere as condições $(\mathrm{KKT})$ do problema $\left(\mathrm{D}_{\mu}\right)$ :

$$
\begin{aligned}
& -\left(\begin{array}{l}
\mathrm{b} \\
\mu \mathrm{Z}^{-1} \mathrm{e}
\end{array}\right)+\left(\begin{array}{l}
\mathrm{A} \\
\mathrm{I}
\end{array}\right) \mathrm{x}=0 \\
& \mathrm{~A}^{\mathrm{t}} \mathrm{y}+\mathrm{z}=\mathrm{c}, \mathrm{z}>0
\end{aligned}
$$

ou seja,

$$
\begin{aligned}
& A x=b \\
& x=\mu Z^{-1} e>0 \Leftrightarrow Z x=\mu e \Leftrightarrow X Z e-\mu e=0 \\
& A^{t} y+z=c, z>0
\end{aligned}
$$

o que coincide com o sistema (2.3.6).

A unicidade do sistema (2.3.6) é devido a unicidade da solução de $\left(\mathrm{P}_{\mu}\right)$. Denotaremos o único ponto $(\mathrm{x}, \mathrm{y}, \mathrm{z})$ que satisfaz (2.3.6), para cada $\mu$, por:

$$
\mathrm{w}(\mu)=(x(\mu), y(\mu), z(\mu)), \text { sendo que } \quad w(\mu) \in \stackrel{\circ}{\mathrm{W}}=\stackrel{\circ}{\mathrm{S}} \times \stackrel{\circ}{\mathrm{T}} \text {. }
$$

Definiremos agora o gap de dualidade no ponto $\mathrm{w} \in \stackrel{\circ}{\mathrm{W}}$ como sendo:

$$
g(w)=c^{t} x-b^{t} y
$$

Usando as duas primeiras equações do sistema (2.3.6) temos:

$$
g(w)=c^{t} x-b^{t} y=c^{t} x-x^{t} A^{t} y=c^{t} x-x^{t}(c-z)=x^{t} z
$$

Em vista disso, usando a equação $\left(2.3 .6 \mathrm{c}^{\prime}\right)$, obtemos: 


$$
\mathrm{g}(\mathrm{w})=\mathrm{x}_{1} \mathrm{z}_{1}+\mathrm{x}_{2} \mathrm{z}_{2}+\ldots+\mathrm{x}_{\mathrm{n}} \mathrm{z}_{\mathrm{n}}=\mu+\mu+\ldots+\mu=\mathrm{n} \mu
$$

Quando $\mu$ converge para zero, $g(w)$ converge para zero, e como $x(\mu)$ e $y(\mu)$ são soluções factíveis dos problemas (P) e (D) respectivamente, segue o resultado de R. C. Monteiro e I. Adler [MON89].

Lema 2.3.1: [MON89] Com $\mu \rightarrow 0, \mathrm{x}(\mu)$ converge para a solução ótima do problema (P) e $(\mathrm{y}(\mu), \mathrm{z}(\mu))$ converge para a solução ótima do problema (D).

Uma direção que será usada nos métodos primais-duais é a direção de Newton. Para encontrá-la, usaremos o Método de Newton.

O Método de Newton é uma das técnicas mais usadas para encontrar uma solução de um sistema de equações não-lineares por meio de aproximações sucessivas do sistema de equações lineares.

Seja $F: \Re^{q} \rightarrow \Re^{q}, F \in C^{2}$. O objetivo aqui é resolver $F(w)=0$.

O Método de Newton para um sistema de equações do tipo $F(w)=0$ consiste em tomar a aproximação linear para $F$ usando expansão de série de Taylor no ponto $w^{k}$. Obtemos:

$$
\mathrm{F}\left(\mathrm{w}^{\mathrm{k}}+\Delta \mathrm{w}^{\mathrm{k}}\right) \cong \mathrm{F}\left(\mathrm{w}^{\mathrm{k}}\right)+\nabla \mathrm{F}\left(\mathrm{w}^{\mathrm{k}}\right) \Delta \mathrm{w}^{\mathrm{k}}=0
$$

onde $\nabla \mathrm{F}$ é o jacobiano de F. Assim a direção de Newton é dada por:

$$
\Delta \mathrm{w}^{\mathrm{k}}=-\left[\nabla \mathrm{F}\left(\mathrm{w}_{\mathrm{k}}\right)\right]^{-1} \mathrm{~F}\left(\mathrm{w}_{\mathrm{k}}\right)
$$

e o método de Newton definido por:

$$
w^{k+1}=w^{k}+\Delta w^{k}
$$

Quando $\nabla \mathrm{F}\left(\mathrm{w}^{*}\right)$ é não-singular e o ponto inicial $\mathrm{w}^{0}$ está próximo da solução $\mathrm{w}^{*}$ do sistema, o teorema a seguir garante convergência quadrática.

Teorema 2.3.4: (Convergência quadrática) Sejam $F: \Re^{q} \rightarrow \Re^{q}, \quad F \in C^{2}$, w ${ }^{*}$ solução do sistema $\mathrm{F}(\mathrm{w})=0, \nabla \mathrm{F}\left(\mathrm{w}^{*}\right)$ não-singular. Existe $\mathrm{w}^{0}$ suficientemente próximo a $\mathrm{w}^{*}$ tal que a seqüência gerada pelo Método de Newton:

$$
\mathrm{w}^{\mathrm{k}+1}=\mathrm{w}^{\mathrm{k}}-\left[\nabla \mathrm{F}\left(\mathrm{w}^{\mathrm{k}}\right)\right]^{-1} \mathrm{~F}\left(\mathrm{w}^{\mathrm{k}}\right)
$$


converge quadraticamente para $\mathrm{w}^{*}$.

Esta convergência rápida, como pode ser visto, é um comportamento local, podendo em outros casos, ser lenta ou mesmo divergir.

Depois dessas considerações, vemos que a direção de Newton $\Delta \mathrm{w}^{\mathrm{k}}$ é determinada pelo sistema de equações (2.3.14).

A seguir, no próximo capítulo, estudaremos os métodos básicos de escala-afim primal e dual, utilizando alguns dos conceitos vistos neste capítulo. 


\section{Capítulo 3}

\section{MÉTOdOS DE ESCALA-AFIM PRIMAL E DUAL}

\subsection{Introdução}

Como vimos no Capítulo 1, os métodos de pontos interiores se desenvolveram a partir de 1984, quando da publicação do algoritmo projetivo de N. Karmarkar [KAR84]. Karmarkar provou que sua complexidade era polinomial e anunciou resultados computacionais superiores aos obtidos com o Método Simplex de Dantzig. Este trabalho levou às intensas pesquisas dos Métodos de Pontos Interiores para a Programação Linear (PL).

Entretanto, o algoritmo de Karmarkar em sua versão original é complexo e, posteriormente, foram apresentados algoritmos derivados daquele, em abordagens bem mais simples.

Uma variante do método projetivo de Karmarkar é o Método de Escala-Afim, que utiliza uma transformação afim em detrimento à transformação projetiva.

O algoritmo de escala-afim básico foi inicialmente apresentado por I.I. Dikin em 1967 (ver [FAN93]). Posteriormente, em 1985, em trabalhos independentes, E.R. Barnes e R.J. Vanderbei, M.S. Meketon, e B.A. Freedman propuseram o algoritmo de escala-afim (primal) para solucionar problemas lineares na forma padrão e estabeleceram a prova da convergência do algoritmo (ver [FAN93]). Um algoritmo similar, chamado Algoritmo de Escala-Afim Dual, foi apresentado e implementado por I. Adler, N. Karmarkar, M.G.C. Resende e G. Veiga [ADL89] para solucionar problemas lineares na forma de desigualdade.

Uma outra variante, chamada Algoritmo Primal-Dual ou Algoritmo Path-following, foi apresentado e analisado por R.C. Monteiro, I. Adler e M.C. Resende, bem como por M. Kojima, 
S. Mizuno e A. Yoshise em 1987. Este algoritmo também é classificado de algoritmo de escalaafim primal-dual [FAN93].

Neste capítulo apresentaremos os Métodos de Escala-Afim Primal e Dual, deixando para o capítulo seguinte, a apresentação do Método Primal-Dual.

Usaremos deste capítulo em diante a mesma notação do capítulo 2, salvo especificação em contrário.

\subsection{Método de Escala-Afim Primal}

\subsubsection{Mudança de escala}

Considere o problema linear (PL) no formato padrão:

\section{(P) Minimize $\mathbf{c}^{\mathbf{t}} \mathbf{x}$ sujeito à $\left\{\begin{array}{l}A x=b \\ x \geq 0\end{array}\right.$}

onde A é uma matriz $\mathrm{m} \times \mathrm{n}$ de posto $\mathrm{m}$; b é um vetor de dimensão $\mathrm{m}$; c e $\mathrm{x}$ são vetores de dimensão $n$.

Seja $\mathrm{S}$ o conjunto factível do problema $(\mathrm{P})$, e $\stackrel{\circ}{\mathrm{S}}$ o interior relativo de $\mathrm{S}$, com respeito ao espaço afim $\{x \mid A x=b\}$, como dados em (2.2.1) e (2.2.2).

Analogamente a (2.2.3) e (2.2.4), a matriz diagonal $\mathrm{n} \times \mathrm{n}$, em relação a $\mathrm{x}^{\mathrm{k}} \in \dot{\mathrm{S}}$, é dada por:

$$
X_{k}=\operatorname{diag}\left(x_{1}^{k}, x_{2}^{k}, \ldots, x_{n}^{k}\right)=\left[\begin{array}{cccc}
x_{1}^{k} & 0 & \cdots & 0 \\
0 & x_{2}^{k} & \cdots & 0 \\
\vdots & \vdots & \ddots & \vdots \\
0 & 0 & \cdots & x_{n}^{k}
\end{array}\right]
$$

A matriz $X_{k}$ é não-singular, com matriz inversa,

$$
\mathrm{X}_{\mathrm{k}}^{-1}=\operatorname{diag}\left(\frac{1}{\mathrm{x}_{1}^{\mathrm{k}}}, \frac{1}{\mathrm{x}_{2}^{\mathrm{k}}}, \ldots, \frac{1}{\mathrm{X}_{\mathrm{n}}^{\mathrm{k}}}\right)=\left[\begin{array}{cccc}
\frac{1}{\mathrm{x}_{1}^{k}} & 0 & \cdots & 0 \\
0 & \frac{1}{\mathrm{x}_{2}^{k}} & \cdots & 0 \\
\vdots & \vdots & \ddots & \vdots \\
0 & 0 & \cdots & \frac{1}{x_{n}^{k}}
\end{array}\right]
$$


Definimos a mudança de variáveis, pela transformação escala-afim, por:

$$
\mathbf{w}=T_{k}(\mathbf{x})=\mathbf{X}_{k}^{-1} \mathbf{x}
$$

Nesta seção 3.2 considere a variável w como acima.

A transformação é bijetora e o problema original (P) na forma escalada corresponde ao seguinte problema de programação linear:

$$
\begin{aligned}
&\left(P_{\text {esc }}\right) \text { Minimize }\left(c^{k}\right)^{t} w \\
& \text { sujeito à }\left\{\begin{array}{l}
A_{k} w=b \\
w \geq 0
\end{array}\right.
\end{aligned}
$$

onde $c^{k}=X_{k} c \quad$ e $\quad A_{k}=A X_{k}$.

Denotamos o conjunto factível de $\left(\mathrm{P}_{\mathrm{esc}}\right)$ por:

$$
S_{\text {esc }}=\left\{w \in \Re_{+}^{n} \mid A_{k} w=b\right\}
$$

A seguir, algumas propriedades da transformação escala-afim.

(T1) $\mathrm{T}_{\mathrm{k}}\left(\mathrm{x}^{\mathrm{k}}\right)=\mathrm{e}$, onde $\mathrm{e}=(1, \ldots, 1)^{\mathrm{t}} \in \mathfrak{R}^{\mathrm{n}}$.

(T2) $T_{k}(x)$ é um vértice de $\left(S_{\text {esc }}\right)$ se, e somente se, $x$ é um vértice de $S$.

(T3) $T_{k}(x)$ está na fronteira de $S_{\text {esc }}$ se, e somente se, $x$ está na fronteira de $S$.

(T4) $\mathrm{T}_{\mathrm{k}}(\mathrm{x})$ é um ponto interior de $\mathrm{S}_{\text {esc }}$ se, e somente se, $\mathrm{x}$ é interior de $\mathrm{S}$.

Note que se $\Delta \mathrm{w}^{\mathrm{k}}$ é uma direção factível para uma escolha apropriada do tamanho do passo $\beta_{\mathrm{k}}>0$, e se o novo ponto $\mathrm{w}^{\mathrm{k}+1}=\mathrm{e}+\beta_{\mathrm{k}} \Delta \mathrm{w}^{\mathrm{k}}$ permanecer no interior da região de factibilidade do problema escalado $S_{\text {esc }}$, a imagem inversa

$$
\mathrm{x}^{\mathrm{k}+1}=\mathrm{T}_{\mathrm{k}}^{-1}\left(\mathrm{w}^{\mathrm{k}+1}\right)=\mathrm{X}_{\mathrm{k}} \mathrm{w}^{\mathrm{k}+1}=\mathrm{x}^{\mathrm{k}}+\beta_{\mathrm{k}}\left(\mathrm{X}_{\mathrm{k}} \Delta \mathrm{w}^{\mathrm{k}}\right)
$$

nos fornecerá uma nova solução factível interior de (P).

A seguir, determinaremos uma direção de busca factível e de descida. 


\subsubsection{Direção}

Para minimizar o valor da função objetivo, adotamos uma direção factível $\Delta \mathrm{w}^{\mathrm{k}}$ de descida.

A direção escolhida é o vetor oposto ao vetor gradiente da função objetivo, projetada no espaço nulo da matriz de restrições $A_{k}$. Neste caso, a matriz de projeção no espaço nulo é dado por:

$$
P_{k}=I-X_{k} A^{t}\left(A X_{k}^{2} A^{t}\right)^{-1} A X_{k}
$$

Logo, a direção de busca é:

$$
\Delta w^{k}=P_{k}\left(-c^{k}\right)=-\left[I-X_{k} A^{t}\left(A X_{k}^{2} A^{t}\right)^{-1} A X_{k}\right] X_{k} c
$$

Entretanto, esta forma dada em (3.2.2.2) não nos é conveniente, devido à necessidade do cálculo da inversa da matriz definida positiva $\left(\mathrm{AX}_{\mathrm{k}}^{2} \mathrm{~A}^{\mathrm{t}}\right)$. O cálculo da direção é melhor arranjado a seguir e o fazemos no espaço das variáveis originais.

Apliquemos a transformação inversa $\mathrm{T}_{\mathrm{k}}^{-1}$ para $\mathrm{w}^{\mathrm{k}+1}$.

$$
\begin{aligned}
& x^{k+1}=T_{k}^{-1}\left(w^{k+1}\right)=X_{k} w^{k+1}=X_{k}\left[e+\beta_{k} \Delta w^{k}\right]= \\
& =x^{k}+\beta_{k} X_{k} \Delta w^{k}=x^{k}+\beta_{k} X_{k}\left(-P_{k} c^{k}\right)= \\
& =x^{k}-\beta_{k} X_{k}\left[I-X_{k} A^{t}\left(A X_{k}^{2} A^{t}\right)^{-1} A X_{k}\right] X_{k} c= \\
& =x^{k}-\beta_{k} X_{k}^{2}\left[c-A^{t}\left(A X_{k}^{2} A^{t}\right)^{-1} A X_{k}^{2} c\right]= \\
& =x^{k}-\beta_{k} X_{k}^{2}\left[c-A^{t} y^{k}\right]
\end{aligned}
$$

onde,

$$
\mathrm{y}^{\mathrm{k}}=\left(\mathrm{AX}_{\mathrm{k}}^{2} \mathrm{~A}^{\mathrm{t}}\right)^{-1} \mathrm{AX} \mathrm{X}_{\mathrm{k}}^{2} \mathrm{c}
$$

é a estimativa dual, cujo valor pode ser calculado por meio do sistema linear, cuja matriz é definida positiva:

$$
\left(A X_{k}^{2} A^{t}\right) y^{k}=A X_{k}^{2} c
$$


Portanto, a direção no espaço de solução original é dado por:

$$
\Delta \mathbf{x}^{\mathrm{k}}=-\mathbf{X}_{\mathbf{k}}^{2}\left[\mathbf{c}-\mathbf{A}^{\mathrm{t}} \mathbf{y}^{\mathbf{k}}\right]
$$

e no espaço transformado é dado por:

$$
\Delta \mathbf{w}^{k}=-\mathbf{X}_{\mathbf{k}}\left[\mathbf{c}-\mathbf{A}^{\mathbf{t}} \mathbf{y}^{\mathbf{k}}\right]
$$

O gap de dualidade do par de soluções factíveis $\left(\mathrm{x}^{\mathrm{k}}, \mathrm{y}^{\mathrm{k}}\right)$ é:

$$
g\left(x^{k}, y^{k}\right)=c^{t} x^{k}-b^{t} y^{k}=e^{t} X_{k}\left[c-A^{t} y^{k}\right]
$$

Caso tenhamos $e^{t} X_{k}\left[c-A^{t} y^{k}\right]=0$, com $\left[c-A^{t} y^{k}\right] \geq 0$, então obtemos a factibilidade primal, a factibilidade dual e as condições das folgas complementares, ou seja, obtemos as condições de Karush-Kuhn-Tucker(KKT). Sendo assim, $x^{k}$ é o ótimo primal e $y^{k}$ o ótimo dual.

$\mathrm{Na}$ próxima seção, obteremos o tamanho do passo a ser dado na direção $\Delta \mathrm{x}^{\mathrm{k}}$, expresso em (3.2.2.6).

\subsubsection{Tamanho do passo}

Trabalhando no espaço de solução transformado, a solução interior $\mathrm{w}^{\mathrm{k}}=\mathrm{e}$, ao longo da direção $\Delta \mathrm{w}^{\mathrm{k}}$, deve fornecer uma nova solução interior $\mathrm{w}^{\mathrm{k}+1}>0$ para que $\mathrm{x}^{\mathrm{k}+1}$ seja interior e possamos repetir o procedimento.

Devemos fazer uma escolha apropriada do tamanho do passo $\beta_{k}>0$, de forma que,

$$
\mathrm{w}^{\mathrm{k}+1}=\mathrm{w}^{\mathrm{k}}+\beta_{\mathrm{k}} \Delta \mathrm{w}^{\mathrm{k}}>0
$$

Caso tenhamos $\Delta \mathrm{w}^{\mathrm{k}} \geq 0$ então $\beta_{\mathrm{k}}$ pode ser qualquer número positivo de forma que obteremos sempre a condição (3.2.3.1). Isto revela um raio de descida e o problema não tem solução ótima finita.

Por outro lado, se $\left(\Delta w^{k}\right)_{i}<0$ para algum i, então teremos:

$$
\mathrm{w}_{\mathrm{i}}^{\mathrm{k}}+\beta_{\mathrm{k}} \Delta \mathrm{w}_{\mathrm{i}}^{\mathrm{k}}>0 \Rightarrow-\beta_{\mathrm{k}} \Delta \mathrm{w}_{\mathrm{i}}^{\mathrm{k}}<\mathrm{w}_{\mathrm{i}}^{\mathrm{k}} \Rightarrow \beta_{\mathrm{k}}<-\frac{\mathrm{w}_{\mathrm{i}}^{\mathrm{k}}}{\Delta \mathrm{w}_{\mathrm{i}}^{\mathrm{k}}}
$$


Observe que $\mathrm{w}^{\mathrm{k}}=\mathrm{e}, \log \mathrm{o}$

$$
\beta_{k}<-\frac{1}{\Delta w_{i}^{k}}
$$

Escolhendo um valor de $\beta_{\mathrm{k}}$ nas condições de (3.2.3.3), podemos nos aproximar demasiadamente da fronteira da região de factibilidade. Para evitá-lo, usamos uma heurística para a escolha do passo.

Tomamos $\alpha, 0<\alpha<1$, e fazemos:

$$
\beta_{k}=\alpha \min \left\{-\frac{1}{\Delta w_{i}^{k}}, \Delta w_{i}^{k}<0, i=1, \ldots, n\right\}
$$

A seguir, após as discussões anteriores, apresentaremos um algoritmo básico.

\subsubsection{Algoritmo de Escala-Afim Primal}

\section{FASE I}

Passo 1 (Inicialização): Seja $\mathrm{k}=0$. Encontre uma solução inicial $\mathrm{x}^{0} \in \stackrel{\circ}{\mathrm{S}}$ (ver seção 3.2.5). Escolha $\alpha$, tal que $0<\alpha<1$ e uma tolerância $\varepsilon>0$.

\section{FASE II}

Passo 2 (Cálculo da estimativa dual): Compute $\left(\mathrm{AX}_{\mathrm{k}}^{2} \mathrm{~A}^{\mathrm{t}}\right) \mathrm{y}^{\mathrm{k}}=\mathrm{AX}_{\mathrm{k}}^{2} \mathrm{c}$.

Passo 3 (Verificação da otimalidade): Calcule $\mathrm{r}^{\mathrm{k}}=\mathrm{c}-\mathrm{A}^{\mathrm{t}} \mathrm{y}^{\mathrm{k}}$.

Se $r^{k} \geq 0$ e $e^{t} X_{k} r^{k} \leq \varepsilon$ então PARE. Resultado: $x^{k}$ é solução ótima primal e $y^{k}$ é solução ótima dual.

Passo 4 (Determinação da direção de busca): Compute $\Delta \mathrm{w}^{\mathrm{k}}=-\mathrm{X}_{\mathrm{k}} \mathrm{r}^{\mathrm{k}}$.

\section{Passo 5 (Verificação da direção de busca):}

Se $\Delta w^{k} \geq 0$ então PARE. Resultado: Problema sem solução ótima finita.

Passo 6 (Cálculo do tamanho do passo): Compute

$$
\beta_{\mathrm{k}}=\alpha \min \left\{-\frac{1}{\Delta \mathrm{w}_{\mathrm{i}}^{\mathrm{k}}}, \Delta \mathrm{w}_{\mathrm{i}}^{\mathrm{k}}<0, \mathrm{i}=1, \ldots, \mathrm{n}\right\}
$$

Passo 7 (Nova solução): Calcule $\Delta \mathrm{x}^{\mathrm{k}}=-\mathrm{X}_{\mathrm{k}}^{2} \mathrm{r}^{\mathrm{k}}$ e $\mathrm{x}^{\mathrm{k}+1}=\mathrm{x}^{\mathrm{k}}+\beta_{\mathrm{k}} \Delta \mathrm{x}^{\mathrm{k}}$.

Faça $\mathrm{k} \leftarrow \mathrm{k}+1$ e siga para o PASSO 2. 


\subsubsection{Solução Inicial (Fase I)}

Nesta seção introduzimos um processo para determinar uma solução inicial para o problema Escala-Afim Primal [FAN93].

O método que será apresentado é o Método M-Grande. Este método é um dos mais facilmente implementados e é apropriado para a maioria das aplicações.

Acrescentamos uma variável $\mathrm{x}^{\mathrm{a}}$, associada a um número $\mathrm{M}$ grande positivo, para $\mathrm{o}$ programa linear original, fazendo com que $(1, \ldots, 1) \in \Re^{\mathrm{n}+1}$ seja uma solução factível interior inicial para o seguinte problema:

\begin{tabular}{|c|c|}
\hline$\left(\mathbf{P}_{\mathbf{M}}\right)$ & 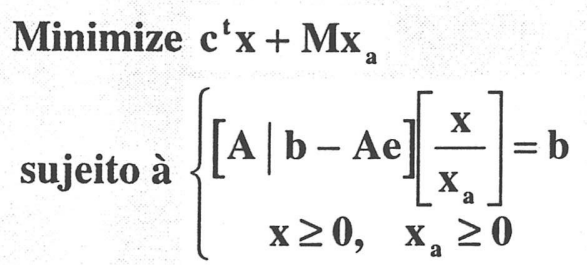 \\
\hline
\end{tabular}

Desta forma temos $n+1$ variáveis.

Aplicamos o Método de Escala-Afim para o problema $\left(\mathrm{P}_{\mathrm{M}}\right)$, com $\mathrm{M}$ suficientemente grande. Se o problema é factível encontraremos a solução ótima do problema $\left(\mathrm{P}_{\mathrm{M}}\right)$ ou concluímos que o problema é ilimitado.

Se a variável artificial $x_{a}$ continuar positiva na solução final $\left(x^{*}, x_{a}^{*}\right)$ o problema original é infactível.

$\mathrm{Se}$, por outro lado, $\mathrm{x}_{\mathrm{a}}$ for nulo, o problema original é ilimitado inferiormente ou $\mathrm{x}^{*}$ soluciona o problema original.

$\mathrm{Na}$ próxima seção estudaremos o algoritmo de escala-afim dual de I. Adler, N. Karmarkar, M. G. C. Resende e G. Veiga [ADL89], que explora a estrutura de desigualdade.

\subsection{Método de Escala-Afim Dual}

\subsubsection{Mudança de escala}

Considere o problema dual de (P): 


\section{(D) Maximize $\mathbf{b}^{\mathbf{t}} \mathbf{y}$ sujeito à $\left\{\begin{array}{c}A^{t} y+z=c \\ z \geq 0\end{array}\right.$ com $y$ irrestrito}

Seja $\mathrm{T}$ o conjunto das soluções factíveis do problema (D), e $\stackrel{\circ}{\mathrm{T}}$ o interior relativo de $\mathrm{T}$ em relação ao espaço afim $\left\{(y, z) \mid A^{t} y+z=c\right\}$, como dado em (2.2.13) e (2.2.14).

Considere a matriz diagonal relacionada à variável de folga $\mathrm{z}^{\mathrm{k}}$, dada por

$$
Z_{k}=\operatorname{diag}\left(z^{k}\right)=\left[\begin{array}{ccc}
z_{1}^{k} & 0 & 0 \\
0 & \ddots & 0 \\
0 & 0 & z_{n}^{k}
\end{array}\right]
$$

Definimos a seguinte mudança de escala pela transformação afim,

$$
\mathbf{w} \in \Re^{\mathrm{m}} \text { tal que } \mathbf{w}=\mathbf{T}_{\mathrm{k}}(\mathrm{z})=\mathbf{Z}_{\mathrm{k}}^{-1} \mathbf{z}
$$

Nesta seção 3.3 considere a variável w como acima.

A transformação é bijetora e o problema original (D) corresponde ao seguinte problema linear:

$$
\begin{array}{ll}
\left(D_{\text {esc }}\right) & \text { Maximize } b^{t} y \\
\text { sujeito à }\left\{\begin{array}{c}
A^{t} y+Z_{k} w=c \\
w \geq 0
\end{array}\right.
\end{array}
$$

Denotamos o conjunto factível de $\left(\mathrm{D}_{\text {esc }}\right)$ por:

$$
\mathrm{T}_{\text {esc }}=\left\{(\mathrm{y}, \mathrm{w}) \in \mathfrak{R}^{\mathrm{m}} \mathrm{X} \mathfrak{R}_{+}^{\mathrm{n}} \mid \mathrm{A}^{\mathrm{t}} \mathrm{y}+\mathrm{Z}_{\mathrm{k}} \mathrm{w}=\mathrm{c}\right\}
$$

A seguir, algumas considerações relacionadas à transformação afim, similares às apresentada na seção 3.2.

(T1) $\mathrm{T}_{\mathrm{k}}\left(\mathrm{z}^{\mathrm{k}}\right)=\mathrm{e}$, onde $\mathrm{e}=(1, \ldots, 1)^{\mathrm{t}} \in \Re^{\mathrm{n}}$.

(T2) $\left(y, T_{k}(z)\right)$ é um vértice de $T_{e s c}$ se, e somente se, $(y, z)$ é um vértice de $T$.

(T3) $\left(\mathrm{y}, \mathrm{T}_{\mathrm{k}}(\mathrm{z})\right)$ está na fronteira de $\mathrm{T}_{\mathrm{esc}}$ se $(\mathrm{y}, \mathrm{z})$ está na fronteira de $\mathrm{T}$.

(T4) $\left(y, T_{k}(z)\right)$ é um ponto interior de $\mathrm{T}_{\text {esc }}$ se $(y, z)$ é interior de $\mathrm{T}$. 
Observe que se $\left(\Delta y^{k}, \Delta z^{k}\right)$ é uma direção factível para uma escolha apropriada do tamanho do passo $\alpha_{k}>0$, e a nova iteração fornecer um ponto interior para $T_{\text {esc }}$, então a imagem inversa fornecerá uma nova solução factível interior de T. Observe também que existe uma correspondência entre os seguintes conjuntos:

$$
\begin{aligned}
& \bar{Y}=\left\{y \in \Re^{m} \mid A^{t} y \leq c\right\} \\
& \bar{W}=\left\{w \in \Re_{+}^{n} \mid \exists y \in \bar{Y} \text { tal que } A^{t} y+Z_{k} w=c\right\}
\end{aligned}
$$

de modo que,

$$
\begin{aligned}
& w(y)=Z_{k}^{-1}\left(c-A^{t} y\right) \\
& y(w)=\left(A Z_{k}^{-2} A^{t}\right)^{-1} A Z_{k}^{-1}\left(Z_{k}^{-1} c-w\right)
\end{aligned}
$$

onde $\mathrm{AZ}_{\mathrm{k}}^{-2} \mathrm{~A}^{\mathrm{t}}$ é matriz simétrica definida positiva, visto que $\mathrm{A}$ tem posto máximo.

A seguir determinaremos uma direção de busca factível e de subida.

\subsubsection{Direção}

Seja $\left(\Delta y^{k}, \Delta z^{k}\right)$ uma direção de subida para o problema original (D) e $\left(\Delta y^{k}, \Delta w^{k}\right)$ a correspondente direção de subida para o problema escalado $\left(\mathrm{D}_{\text {esc }}\right)$.

Note que existe uma correspondência entre $\Delta y^{k}$ e $\Delta w^{k}$ como entre $y^{k}$ e w ${ }^{k}$. Para expressar esta correspondência, observe que a direção factível para o problema $\left(D_{\text {esc }}\right)$ deve pertencer ao núcleo de $\left(\mathrm{A}^{\mathrm{t}}, \mathrm{Z}_{\mathrm{k}}\right)$ e, por conseguinte, $\Delta \mathrm{w}$, deve pertencer ao espaço imagem de $\mathrm{Z}_{\mathrm{k}}^{-1} \mathrm{~A}^{\mathrm{t}}$. Disto obtemos:

$$
\begin{aligned}
& \mathrm{Z}_{\mathrm{k}}^{-1} \mathrm{~A}^{\mathrm{t}} \Delta \mathrm{y}^{\mathrm{k}}+\Delta \mathrm{w}^{\mathrm{k}}=0 \\
& \mathrm{AZ}_{\mathrm{k}}^{-1}\left[\mathrm{Z}_{\mathrm{k}}^{-1} \mathrm{~A}^{\mathrm{t}} \Delta \mathrm{y}^{\mathrm{k}}+\Delta \mathrm{w}^{\mathrm{k}}\right]=0 \\
& \Delta \mathrm{y}^{\mathrm{k}}=-\left(\mathrm{AZ}_{\mathrm{k}}^{-2} \mathrm{~A}^{\mathrm{t}}\right)^{-1} \mathrm{AZ}_{\mathrm{k}}^{-1} \Delta \mathrm{w}^{\mathrm{k}}
\end{aligned}
$$

ou seja,

$$
\Delta \mathrm{w}^{\mathrm{k}}=-\mathrm{Z}_{\mathrm{k}}^{-1} \mathrm{~A}^{\mathrm{t}} \Delta \mathrm{y}^{\mathrm{k}} \quad \text { e } \quad \Delta \mathrm{y}^{\mathrm{k}}=-\left(\mathrm{AZ}_{\mathrm{k}}^{-2} \mathrm{~A}^{\mathrm{t}}\right)^{-1} \mathrm{~A} \mathrm{Z}_{\mathrm{k}}^{-1} \Delta \mathrm{w}^{\mathrm{k}}
$$


Observada estas condições, o método adota a direção de busca $\left(\Delta \mathrm{y}^{\mathrm{k}}, \Delta \mathrm{w}^{\mathrm{k}}\right)$ como sendo a direção do gradiente da função objetivo projetada no espaço de factibilidade, com respeito às variáveis escaladas. Como somente as variáveis de folga são alteradas pela transformação afim, computamos o gradiente da função objetivo com respeito a w por:

$$
\nabla_{w}\left(b^{t} y(w)\right)=-b^{t}\left(A Z_{k}^{-2} A^{t}\right)^{-1} A Z_{k}^{-1}=-Z_{k}^{-1} A^{t}\left(A Z_{k}^{-2} A^{t}\right)^{-1} b
$$

Como o gradiente já se encontra no espaço-imagem de $\mathrm{Z}_{\mathrm{k}}^{-1} \mathrm{~A}^{\mathrm{t}}$, torna-se desnecessária a projeção sobre esse espaço, logo a direção de busca em $\overline{\mathrm{W}}$ é dado por:

$$
\Delta \mathbf{w}^{k}=-\mathbf{Z}_{k}^{-1} \mathbf{A}^{t}\left(\mathbf{A} \mathbf{Z}_{k}^{-2} \mathbf{A}^{t}\right)^{-1} \mathbf{b}
$$

e por (3.3.2.1) temos a direção de busca em $\bar{Y}$ procurada,

$$
\Delta \mathbf{y}^{\mathrm{k}}=\left(\mathbf{A} \mathbf{Z}_{\mathrm{k}}^{-2} \mathbf{A}^{\mathrm{t}}\right)^{-1} \mathbf{b}
$$

Aplicando a transformação afim inversa para $\Delta \mathrm{w}^{\mathrm{k}}$ obteremos a correspondente direção factível para as folgas não-escaladas $\mathrm{z}^{\mathrm{k}}$, de modo que,

$$
\Delta \mathbf{z}^{k}=-\mathbf{A}^{t} \Delta \mathbf{y}^{k}
$$

Observe que de (3.3.2.4) obtemos:

$$
\left(\mathrm{AZ}_{\mathrm{k}}^{-2} \mathrm{~A}^{\mathrm{t}}\right) \Delta \mathrm{y}^{\mathrm{k}}=\mathrm{b}=\mathrm{Ax^{ \textrm {k } }}
$$

Logo uma estimativa primal, análogo ao procedimento da estimativa dual, é dado por:

$$
\mathrm{x}^{\mathrm{k}}=\mathrm{Z}_{\mathrm{k}}^{-2} \mathrm{~A}^{\mathrm{t}} \Delta \mathrm{y}^{\mathrm{k}}=-\mathrm{Z}_{\mathrm{k}}^{-2} \Delta \mathrm{z}^{\mathrm{k}}
$$

Encontrada as direções, (3.3.2.3)-(3.3.2.5) calcularemos na próxima seção o tamanho do passo $\alpha_{\mathrm{k}}$ a ser dado.

\subsubsection{Tamanho do passo}

Analogamente à escolha do tamanho do passo na seção (3.2.3) para o método primal, dado uma solução $\left(\mathrm{y}^{\mathrm{k}}, \mathrm{z}^{\mathrm{k}}\right)$, ao longo da direção $\left(\Delta \mathrm{y}^{\mathrm{z}}, \Delta \mathrm{z}^{\mathrm{k}}\right)$, a nova iteração deve ser interior. 
Para tanto, seja $\alpha^{\mathrm{k}}>0$ e,

$$
\begin{aligned}
& \mathrm{y}^{\mathrm{k}+1}=\mathrm{y}^{\mathrm{k}}+\alpha_{\mathrm{k}} \Delta \mathrm{y}^{\mathrm{k}} \\
& \mathrm{z}^{\mathrm{k}+1}=\mathrm{z}^{\mathrm{k}}+\alpha_{\mathrm{k}} \Delta \mathrm{z}^{\mathrm{k}}
\end{aligned}
$$

Como $\mathrm{y}^{\mathrm{k}}$ é irrestrito, consideraremos somente a variável $\mathrm{z}^{\mathrm{k}}$. Para $\mathrm{z}^{\mathrm{k}+1}$ ser interior, devemos ter:

$$
\mathrm{z}^{\mathrm{k}}+\alpha_{\mathrm{k}} \Delta \mathrm{z}^{\mathrm{k}}>0
$$

Caso tenhamos $\Delta \mathrm{z}^{\mathrm{k}} \geq 0$ a solução é ilimitada, pois qualquer $\alpha_{\mathrm{k}}$ positivo torna verdadeiro (3.3.3.2).

Entretanto, se $\Delta z_{i}^{k}<0$ para algum i, então teremos:

$$
z_{i}^{k}+\alpha_{k} \Delta z_{i}^{k}>0 \Rightarrow \alpha_{k}<-\frac{z_{i}^{k}}{\Delta z_{i}^{k}}
$$

Para evitar que um valor de $\alpha_{\mathrm{k}}$ faça com que nos aproximemos muito da fronteira, usaremos a seguinte heurística: tomamos $0<\varepsilon<1$ e fazemos,

$$
\alpha_{k}=\varepsilon \min \left\{-\frac{z_{i}^{k}}{\Delta z_{i}^{k}}, \quad \Delta z_{i}^{k}<0, \quad i=1, \ldots, n\right\}
$$

A seguir, dada as condições apresentadas nas seções anteriores, podemos estruturar o algoritmo de escala-afim dual.

\subsubsection{Algoritmo de Escala-Afim Dual}

\section{FASE I}

Passo 1 (Inicialização): Seja $k=0$. Encontre uma solução inicial $y^{0} \in \stackrel{\circ}{T}$ (ver seção 3.3.5). Faça $\mathrm{z}^{0}=\mathrm{c}-\mathrm{A}^{\mathrm{t}} \mathrm{y}^{0}$ e escolha $\varepsilon$ tal que $0<\varepsilon<1$.

\section{FASE II}

Passo 2 (Determinação da direção de busca): Calcule

$$
\begin{aligned}
& \left(\mathrm{AZ}_{\mathrm{k}}^{-2} \mathrm{~A}^{\mathrm{t}}\right) \Delta \mathrm{y}^{\mathrm{k}}=\mathrm{b}, \text { onde } \mathrm{Z}_{\mathrm{k}}=\operatorname{diag}\left[\mathrm{z}_{1}^{\mathrm{k}}, \ldots, \mathrm{z}_{\mathrm{n}}^{\mathrm{k}}\right] \\
& \Delta \mathrm{z}^{\mathrm{k}}=-\mathrm{A}^{\mathrm{t}} \Delta \mathrm{y}^{\mathrm{k}}
\end{aligned}
$$

\section{Passo 3 (Verificação da direção de busca):}

Se $\Delta \mathrm{z}^{\mathrm{k}} \geq 0$ então PARE. Resultado: Problema sem solução ótima finita. 
Passo 4 (Cálculo da estimativa primal): Seja $x^{k}=-Z_{k}^{-2} \Delta z^{k}$

Passo 5 (Verificação da otimalidade): Se $x^{k} \geq 0$ e $c^{t} x^{k}-b^{t} y^{k} \leq \lambda, \lambda$ escalar positivo pequeno, então PARE. Resultado: $\left(\mathrm{y}^{\mathrm{k}}, \mathrm{z}^{\mathrm{k}}\right)$ é ótimo dual e $\mathrm{x}^{\mathrm{k}}$ é ótimo primal.

Passo 6 (Cálculo do tamanho do passo): Calcule

$$
\begin{aligned}
& \alpha_{k}=\min \left\{-\frac{z_{i}^{k}}{\Delta z_{i}^{k}}, \quad \Delta z_{i}^{k}<0, \quad i=1, \ldots, n\right\} \\
& \alpha_{k}=\varepsilon \alpha_{k}
\end{aligned}
$$

Passo 7 (Nova solução): Calcule

$$
\begin{aligned}
& \mathrm{y}^{\mathrm{k}+1}=\mathrm{y}^{\mathrm{k}}+\alpha_{\mathrm{k}} \Delta \mathrm{y}^{\mathrm{k}} \\
& \mathrm{z}^{\mathrm{k}+1}=\mathrm{z}^{\mathrm{k}}+\alpha_{\mathrm{k}} \Delta \mathrm{z}^{\mathrm{k}}\left(\text { ou } \mathrm{z}^{\mathrm{k}+1}=\mathrm{c}-\mathrm{A}^{\mathrm{t}} \mathrm{y}^{\mathrm{k}+1}\right)
\end{aligned}
$$

Faça $\mathrm{k} \leftarrow \mathrm{k}+1$ e siga para o PASSO 2 .

\subsubsection{Solução Inicial (Fase I)}

Nesta seção apresentaremos o Método M-Grande para obtenção da solução inicial do problema de Escala-Afim Dual [ADL89].

Note que, se c > 0 então obtemos uma solução factível interior imediata para o problema (D), escolhendo $\mathrm{y}^{0}=0 \mathrm{e} \mathrm{z}^{0}=\mathrm{c}$. Entretanto este é um caso especial. Na maioria das vezes utilizamos recursos como o método M-Grande para a inicialização do problema (D).

O procedimento para o método M-Grande é similar ao apresentado na seção 3.2.5. Escolha:

$$
y^{0}=\left(\frac{\|c\|}{\left\|A^{t} b\right\|}\right) b
$$

Se $z^{0}=c-A^{t} y^{0}>0$ então $y^{0}$ é ponto interior factível inicial.

Caso contrário, acrescente uma variável artificial y tal que,

$$
y_{a}^{0}=-2 \min \left\{\left(c-A^{t} y^{0}\right)_{i}, i=1, \ldots, n\right\}
$$

Embora pouco frequente, é possível que $\mathrm{y}_{\mathrm{a}}^{0} \approx 0$. Neste caso, o melhor é tomarmos:

$$
y_{a}^{0}=2\left\|c-A^{t} y^{0}\right\|
$$


$\mathrm{O}$ vetor $\left(\mathrm{y}^{0}, \mathrm{y}_{\mathrm{a}}^{0}, \mathrm{z}^{0}\right) \in \mathfrak{R}^{\mathrm{m}} \times \mathfrak{R} \times \mathfrak{R}^{\mathrm{n}}$ é uma solução factível interior inicial da Fase I do problema dado a seguir:

\begin{tabular}{|lc|}
\hline$\left(D_{M}\right)$ & Maximize $b^{t} y-M y_{a}$ \\
& sujeito à $\left\{\begin{array}{c}A^{t} y-e y_{a}+z=c \\
z \geq 0\end{array}\right.$ \\
\hline
\end{tabular}

onde $\quad \mathrm{M}=\xi \mathrm{b}^{\mathrm{t}} \frac{\mathrm{y}^{0}}{\mathrm{y}_{\mathrm{a}}^{0}}, \xi$ grande.

Aplicamos o Método Escala-Afim Dual no problema $\left(D_{M}\right) . O$ algoritmo identifica uma solução factível dual interior $\left(\mathrm{y}^{*}, \mathrm{y}_{\mathrm{a}}{ }^{*}, \mathrm{z}^{*}\right)$ ou , se a solução não existir, encontra uma solução $\left(\mathrm{y}^{*}, \mathrm{y}_{\mathrm{a}}^{*}, \mathrm{z}^{*}\right)$ que satisfaz o critério de parada do problema original. Com $\varepsilon_{\mathrm{f}}$ definido como uma tolerância de factibilidade, a modificação do critério de parada para a FASE 1 é formulada como se segue:

a) Se $y_{a}^{k}<0$ para uma iteração $k$, então $y^{k}$ é uma solução factível interior para o problema (D).

b) Se $\mathrm{y}_{\mathrm{a}}^{\mathrm{k}}>\varepsilon_{\mathrm{f}}$ e o algoritmo satisfaz um critério de parada, então o problema original é declarado infactível.

c) $\operatorname{Se} y_{\mathrm{a}}^{\mathrm{k}}<\varepsilon_{\mathrm{f}}$ e o algoritmo satisfaz um critério de parada, ou o problema original é ilimitado ou a solução ótima é encontrada. Neste caso, (D) não tem solução factível interior.

Se a FASE 1 termina de acordo com a condição a), o algoritmo é aplicado ao problema original (D) inicializando com a última iteração da FASE 1 e usando um critério de parada regular.

NOTA: Como observação, citamos que a estrutura de desigualdade $\mathrm{A}^{\mathrm{t}} \mathrm{y} \leq \mathrm{c}$, explorada pelo algoritmo escala-afim dual, permite que se estenda o método visto para problemas lineares com restrições canalizadas do tipo $\mathrm{c}^{-} \leq \mathrm{A}^{t} \mathrm{y} \leq \mathrm{c}^{+}$, sendo esta uma decorrência imediata, com uma ligeira modificação do mesmo (ver [ARE90], [BAL91]).

No próximo capítulo estudaremos um algoritmo básico primal-dual. 


\section{MÉTODO PRIMAL-DUAL}

\subsection{Introdução}

Além dos métodos de escala-afim outros métodos foram desenvolvidos, como por exemplo, método primal de centros, métodos primais-duais de redução de potencial, métodos primais com função penalizada, métodos primais-duais com função penalizada, entre outros.

Praticamente todos os métodos de pontos interiores que buscavam um melhor desempenho do ponto de vista da complexidade tinham a preocupação em manter-se longe da fronteira da região de factibilidade. Para isso uma ferramenta, estudada inicialmente por K. R. Frisch [FRI55] em 1955, tornou-se importante, a Função Barreira Logarítmica:

$$
f(x)=-\sum_{i=1}^{n} \log x_{i}, \quad x \in \Re^{n}, \quad x>0
$$

Esta função cresce indefinidamente quando nos aproximamos da fronteira da região factível, ou seja, quando alguma coordenada $x_{i}$ tende a zero.

Muitos métodos procuram reduzir os custos utilizando-se dessa função barreira e tentam percorrer pelo "meio" da região factível. Um meio viável é "caminhar" pela curva denominada trajetória central(a ser definido posteriormente).

Métodos que buscam seguir a trajetória central são chamados por muitos pesquisadores de métodos path-following [GON92][MON89][etc.]. 
J. Renegar [REN88] foi o primeiro a obter redução da complexidade da função centro(método primal de centros) em detrimento à função potencial de Karmarkar, seguindo de perto a trajetória central.

P. E. Gill, W. Murray, M. A. Saunders, J. A. Tomlin e M. H. Wright usaram o método da função barreira logarítmica para desenvolver o método de barreira de Newton projetada e mostraram uma equivalência para o algoritmo de escala projetiva de Karmarkar em 1985 (ver [FAN93]). N. Megiddo propôs uma estrutura primal-dual em 1986 (ver [FAN93]). Usando essa estrutura, M. Kojima, S. Mizuno e A. Yoshise apresentaram um algoritmo primal-dual de tempo polinomial para problemas de programação linear em 1987. A complexidade era de $\mathrm{O}\left(\mathrm{n}^{4} \mathrm{~L}\right)$ operações aritméticas (ver [FAN93]). Mais tarde, R. C. Monteiro e I. Adler melhoraram o algoritmo primal-dual para convergir em no máximo $\mathrm{O}(\sqrt{\mathrm{n} L})$ iterações com $\mathrm{O}\left(\mathrm{n}^{2.5}\right)$ operações aritméticas por iteração, resultando em um total de $\mathrm{O}\left(\mathrm{n}^{3} \mathrm{~L}\right)$ operações aritméticas [MON89][MON89-2].

Neste capítulo apresentaremos um método bastante estudado atualmente, segundo bibliografia consultada, o Método Primal-Dual. Por procurar seguir a trajetória central é também classificado como Método Path-following, além de ser denominado de Método de Escala-Afim Primal-Dual [FAN93].

O algoritmo primal-dual básico de pontos interiores para a programação linear(PL) a cada iteração utilizará um parâmetro de penalização e encontrará a direção de Newton associada ao sistema de equações de Karush-Kuhn-Tucker (KKT) que caracteriza a solução do problema de função barreira logarítmica. O método é baseado na idéia de perseguir a trajetória descrita pelas soluções obtidas pela variação do parâmetro de penalização.

\subsection{Trajetória central}

Considere o seguinte problema linear no formato padrão:

\begin{tabular}{|ll|}
\hline (P) & Minimize $\mathbf{c}^{\mathbf{t}} \mathbf{x}$ \\
& sujeito à $\left\{\begin{array}{l}\mathbf{A x}=\mathbf{b} \\
\mathbf{x} \geq \mathbf{0}\end{array}\right.$ \\
\hline
\end{tabular}

e seu dual: 


\section{(D) Maximize $b^{t} \mathbf{y}$ \\ sujeito à $\left\{\begin{array}{l}A^{t} y+z=c \\ z \geq 0\end{array}\right.$ com $y$ irrestrito,}

onde A é uma matriz $\mathrm{m} \times \mathrm{n}$ e b,c são vetores de dimensões $\mathrm{m}$ e $\mathrm{n}$, respectivamente.

Note que, para $x>0$, podemos aplicar a técnica da função barreira logarítmica em (P) e considerar a seguinte família de problemas de programação não-linear:

$$
\begin{array}{r}
\left(\mathbf{P}_{\mu}\right) \text { Minimize } c^{\mathbf{t} x}-\mu \sum_{j=1}^{n} \ln \left(\mathbf{x}_{\mathrm{j}}\right) \\
\text { sujeito à }\left\{\begin{array}{l}
\mathbf{A x}=\mathbf{b} \\
\mathbf{x}>0
\end{array}\right.
\end{array}
$$

onde $\mu>0$ é um parâmetro de barreira ou penalidade. Quando $\mu$ decresce para zero, a solução ótima de $\left(\mathrm{P}_{\mu}\right)$ converge para a solução ótima do problema de programação linear original (P), conforme Lema 2.3.1, na seção 2.3.

A unicidade da solução do problema $\left(\mathrm{P}_{\mu}\right)$ para cada $\mu>0$ decorre do fato de o problema $\left(\mathrm{P}_{\mu}\right)$ ser estritamente convexo, o que satisfaz as hipóteses do Teorema 2.3.3.

Tomando por base as condições KKT, aplicado ao problema $\left(\mathrm{P}_{\mu}\right)$, e introduzindo o vetor $z=\mu X^{-1} e$, obtemos o seguinte sistema, visto na seção 2.3:

$$
\begin{array}{ll}
\mathrm{Ax}=\mathrm{b}, \mathrm{x}>0 & \text { (factibilidade primal) } \\
\mathrm{A}^{\mathrm{t}} \mathrm{y}+\mathrm{z}=\mathrm{c} & \text { (factibilidade dual) } \\
\mathrm{XZe}-\mu \mathrm{e}=0 & \text { (folgas complementares) }
\end{array}
$$

onde $\mathrm{Z}=\operatorname{diag}\left(\mathrm{z}_{1}, \ldots, \mathrm{z}_{\mathrm{n}}\right)$ e $\mathrm{z}=\mathrm{Ze}$.

O sistema (4.2.1) caracteriza a solução do problema $\left(P_{\mu}\right)$ e também a solução do seguinte problema:

$$
\begin{aligned}
& \left(D_{\mu}\right) \text { Maximize } \mathbf{b}^{t} \mathbf{y}+\mu \sum_{\mathrm{j}=1}^{\mathrm{n}} \ln \left(\mathrm{z}_{\mathrm{j}}\right) \\
& \text { sujeito à }\left\{\begin{array}{l}
\mathbf{A}^{\mathrm{t}} \mathrm{y}+\mathrm{z}=\mathrm{c} \\
\mathrm{z}>0
\end{array},\right. \text { y irrestrito }
\end{aligned}
$$


Tal afirmação está melhor desenvolvida na seção 2.3.

$\mathrm{O}$ único ponto $(\mathrm{x}, \mathrm{y}, \mathrm{z})$ satisfazendo $(4.2 .1)$, para cada $\mu$, é denotado por $\mathrm{w}(\mu)=(\mathrm{x}(\mu), \mathrm{y}(\mu), \mathrm{z}(\mu)), \mathrm{w}(\mu) \in \stackrel{\circ}{\mathrm{W}}=\stackrel{\circ}{\mathrm{S}} \mathrm{x} \stackrel{\circ}{\mathrm{T}}$.

Para $\mu>0$, denotaremos por $\Gamma$ a curva (ou trajetória) consistindo das soluções $w(\mu)$ para o sistema (4.2.1), isto é,

$$
\Gamma=\{\mathrm{w}(\mu)=(\mathrm{x}(\mu), \mathrm{y}(\mu), \mathrm{z}(\mu)) ; \mu>0, \text { tal que satisfaça o sistema (4.2.1) }\}
$$

A trajetória $\Gamma$ é usualmente referida como a Trajetória Central associada ao problema (P). Alguns autores (por exemplo [GON92]) preferem definir a trajetória central apenas como o trajeto descrito pelas variáveis primais $\mathrm{x}(\mu)$.

O algoritmo primal-dual é baseado na idéia de buscar as soluções dos problemas (P) e (D) aproximando a trajetória central $\Gamma$. A trajetória $\Gamma$ servirá como um guia que direcionará os pontos gerados pelo algoritmo.

Em termos de implementação, o algoritmo primal-dual trabalha com uma aproximação da solução do sistema (4.2.1) para cada $\mu>0$, com $\mu$ sendo decrementado iterativamente e aproximando-se de zero.

A seguir apresentamos como um método para resolver o sistema (4.2.1) produz uma direção de busca.

\subsection{Direção de Newton}

Dado um ponto inicial $\mathrm{w}^{0}=\left(\mathrm{x}^{0}, \mathrm{y}^{0}, \mathrm{z}^{0}\right) \in \stackrel{\circ}{\mathrm{S}} \times \stackrel{\circ}{\mathrm{T}}$, o algoritmo primal-dual gera uma seqüência de pontos $\left\{\mathrm{w}^{\mathrm{k}+1}=\mathrm{w}^{\mathrm{k}}+\beta_{\mathrm{k}} \Delta \mathrm{w}^{\mathrm{k}} \in \stackrel{\circ}{\mathrm{S}} \times \stackrel{\circ}{\mathrm{T}}\right\}$, onde $\Delta \mathrm{w}^{\mathrm{k}}$ é a direção de busca e $\beta_{\mathrm{k}} \mathrm{o}$ passo a cada iteração.

Tal direção $\Delta \mathrm{w}^{\mathrm{k}}=\left(\Delta \mathrm{x}^{\mathrm{k}}, \Delta \mathrm{y}^{\mathrm{k}}, \Delta \mathrm{z}^{\mathrm{k}}\right)$, que gera o próximo ponto da iteração, é dada pela direção de Newton associada ao sistema de equações (KKT) (4.2.1).

Seja:

$$
F(w)=\left[\begin{array}{l}
A x-b \\
A^{t} y+z-c \\
X Z e-\mu e
\end{array}\right], \text { onde } w=(x, y, z)
$$

O sistema (4.2.1) é então: $F(w)=0$. 
O Método de Newton aplicado ao sistema de equações (4.2.1) a partir de uma aproximação $w^{k}$ nos fornece a direção de Newton $\Delta w^{k}$, que é determinada pelo seguinte sistema de equações lineares:

$$
\mathrm{F}\left(\mathrm{w}^{\mathrm{k}}\right)+\nabla \mathrm{F}\left(\mathrm{w}^{\mathrm{k}}\right) \Delta \mathrm{w}^{\mathrm{k}}=0
$$

que com as devidas substituições nos fornece:

$$
\left[\begin{array}{c}
\mathrm{Ax}^{\mathrm{k}}-\mathrm{b} \\
\mathrm{A}^{\mathrm{t}} \mathrm{y}^{\mathrm{k}}+\mathrm{z}^{\mathrm{k}}-\mathrm{c} \\
\mathrm{X}_{\mathrm{k}} \mathrm{Z}_{\mathrm{k}} \mathrm{e}-\mu \mathrm{e}
\end{array}\right]+\left[\begin{array}{ccc}
\mathrm{A} & 0 & 0 \\
0 & \mathrm{~A}^{\mathrm{t}} & \mathrm{I} \\
\mathrm{Z}_{\mathrm{k}} & 0 & \mathrm{X}_{\mathrm{k}}
\end{array}\right]\left[\begin{array}{c}
\Delta \mathrm{x}^{\mathrm{k}} \\
\Delta \mathrm{y}^{\mathrm{k}} \\
\Delta \mathrm{z}^{\mathrm{k}}
\end{array}\right]=\left[\begin{array}{l}
0 \\
0 \\
0
\end{array}\right]
$$

Fazendo algumas operações simples, obtemos de (4.3.3) as seguintes equações:

$$
\begin{aligned}
& \mathrm{A} \Delta \mathrm{x}^{\mathrm{k}}=\mathrm{b}-\mathrm{Ax^{k }} \\
& \mathrm{A}^{\mathrm{t}} \Delta \mathrm{y}^{\mathrm{k}}+\Delta \mathrm{z}^{\mathrm{k}}=\mathrm{c}-\mathrm{A}^{\mathrm{t}} \mathrm{y}^{\mathrm{k}}-\mathrm{z}^{\mathrm{k}} \\
& \mathrm{Z}_{\mathrm{k}} \Delta \mathrm{x}^{\mathrm{k}}+\mathrm{X}_{\mathrm{k}} \Delta \mathrm{z}^{\mathrm{k}}=\mu \mathrm{e}-\mathrm{X}_{\mathrm{k}} \mathrm{Z}_{\mathrm{k}} \mathrm{e}
\end{aligned}
$$

Para efeito de simplicidade de notação, façamos:

$$
t^{k}=b-A x^{k}, \quad u^{k}=c-A^{t} y^{k}-z^{k} \quad \text { e } \quad v^{k}=\mu e-X_{k} Z_{k} e
$$

Com isso obtemos, por cálculos simples, as seguintes expressões da solução do sistema (4.3.4):

$$
\begin{aligned}
& \Delta x^{k}=Z_{k}^{-1} X_{k} A^{t}\left(A Z_{k}^{-1} X_{k} A^{t}\right)^{-1}\left[t^{k}-A Z_{k}^{-1}\left(v^{k}-X_{k} u^{k}\right)\right]+Z_{k}^{-1}\left(v^{k}-X_{k} u^{k}\right) \\
& \Delta y^{k}=\left(A Z_{k}^{-1} X_{k} A^{t}\right)^{-1}\left[t^{k}-A Z_{k}^{-1}\left(v^{k}-X_{k} u^{k}\right)\right] \\
& \Delta z^{k}=u^{k}-A^{t}\left\{\left(A Z_{k}^{-1} X_{k} A^{t}\right)^{-1}\left[t^{k}-A Z_{k}^{-1}\left(v^{k}-X_{k} u^{k}\right)\right]\right\}
\end{aligned}
$$

Observe que se $\mathrm{x}^{\mathrm{k}} \in \stackrel{\circ}{\mathrm{S}}$ e $\left(\mathrm{y}^{\mathrm{k}}, \mathrm{z}^{\mathrm{k}}\right) \in \stackrel{\circ}{\mathrm{T}}$ então $\mathrm{t}^{\mathrm{k}}=0$ e $\mathrm{u}^{\mathrm{k}}=0$.

Logo, se os pontos gerados pelo método forem pontos factíveis interiores, teremos as seguintes expressões:

$$
\begin{aligned}
\Delta \mathbf{x}^{\mathrm{k}} & =\left[\mathbf{Z}_{\mathrm{k}}^{-1}-\mathbf{Z}_{\mathrm{k}}^{-1} \mathbf{X}_{\mathrm{k}} \mathbf{A}^{\mathrm{t}}\left(\mathbf{A} \mathbf{Z}_{\mathrm{k}}^{-1} \mathbf{X}_{\mathrm{k}} \mathbf{A}^{\mathrm{t}}\right)^{-1} \mathbf{A} \mathbf{Z}_{\mathrm{k}}^{-1}\right] \mathbf{v}^{\mathrm{k}} \\
\Delta \mathbf{y}^{\mathrm{k}} & =-\left(\mathbf{A} \mathbf{Z}_{\mathrm{k}}^{-1} \mathbf{X}_{\mathrm{k}} \mathbf{A}^{\mathrm{t}}\right)^{-1} \mathbf{A} \mathbf{Z}_{\mathrm{k}}^{-1} \mathbf{v}^{\mathrm{k}}
\end{aligned}
$$




$$
\Delta \mathbf{z}^{\mathrm{k}}=\mathbf{A}^{\mathrm{t}}\left(\mathbf{A} \mathbf{Z}_{\mathbf{k}}^{-1} \mathbf{X}_{\mathbf{k}} \mathbf{A}^{\mathrm{t}}\right)^{-1} \mathbf{A} \mathbf{Z}_{\mathbf{k}}^{-1} \mathbf{v}^{\mathrm{k}}
$$

Entretanto, neste caso é necessário encontrar a inversa da matriz definida positiva $\mathrm{AZ}_{\mathrm{k}}^{-1} \mathrm{X}_{\mathrm{k}} \mathrm{A}^{\mathrm{t}}$, o que significa resolver um sistema linear de equações com essa matriz. Esse tipo de resolução é desvantajoso do ponto de vista computacional, uma vez que a esparsidade de A pode ser perdida no processo. Visando evitar essa desvantagem, encontraremos a seguir, outras expressões para a direção de movimento.

Ao invés de explicitarmos as incógnitas do sistema (4.3.4) obtendo a solução em (4.3.6), o que equivale a definir previamente uma ordem na eliminação de Gauss, explicitamos apenas $\Delta \mathrm{z}$ e resolvemos o sistema resultante.

De (4.3.4) temos:

$$
\begin{aligned}
& \mathrm{A} \Delta \mathrm{x}^{\mathrm{k}}=\mathrm{b}-\mathrm{Ax^{ \textrm {k } }} \\
& \mathrm{A}^{\mathrm{t}} \Delta \mathrm{y}^{\mathrm{k}}+\Delta \mathrm{z}^{\mathrm{k}}=\mathrm{c}-\mathrm{A}^{\mathrm{t}} \mathrm{y}^{\mathrm{k}}-\mathrm{z}^{\mathrm{k}} \\
& \Delta \mathrm{z}^{\mathrm{k}}=\mu \mathrm{X}_{\mathrm{k}}^{-1} \mathrm{e}-\mathrm{z}^{\mathrm{k}}-\mathrm{X}_{\mathrm{k}}^{-1} \mathrm{Z}_{\mathrm{k}} \Delta \mathrm{x}^{\mathrm{k}}
\end{aligned}
$$

Substituindo (4.3.8c) em (4.3.8b) obtemos as seguintes equações:

$$
\begin{aligned}
& \mathrm{A} \Delta \mathrm{x}^{\mathrm{k}}=\mathrm{b}-\mathrm{Ax} \mathrm{k}^{\mathrm{k}} \\
& \mathrm{A}^{\mathrm{t}} \Delta \mathrm{y}^{\mathrm{k}}-\mathrm{X}_{\mathrm{k}}^{-1} \mathrm{Z}_{\mathrm{k}} \Delta \mathrm{x}^{\mathrm{k}}=\mathrm{c}-\mathrm{A}^{\mathrm{t}} \mathrm{y}^{\mathrm{k}}-\mu \mathrm{X}_{\mathrm{k}}^{-1} \mathrm{e}
\end{aligned}
$$

Colocando o sistema (4.3.9) na forma matricial, obtemos:

$$
\left[\begin{array}{cc}
-\mathbf{X}_{k}^{-1} \mathbf{Z}_{k} & \mathbf{A}^{\mathrm{t}} \\
\mathbf{A} & 0
\end{array}\right]\left[\begin{array}{c}
\Delta \mathbf{x}^{k} \\
\Delta \mathbf{y}^{k}
\end{array}\right]=\left[\begin{array}{c}
\mathbf{c}-\mathbf{A}^{\mathrm{t}} \mathbf{y}^{\mathrm{k}}-\mu \mathbf{X}_{k}^{-1} \mathbf{e} \\
\mathbf{b}-\mathbf{A} \mathbf{x}^{k}
\end{array}\right]
$$

Desta forma teremos a direção $\Delta \mathbf{w}^{\mathbf{k}}=\left(\Delta \mathbf{x}^{\mathbf{k}}, \Delta \mathbf{y}^{\mathbf{k}}, \Delta \mathbf{z}^{\mathbf{k}}\right)$ de Newton que pode ser obtida resolvendo-se (4.3.10) e a esparsidade original de A estará preservada.

Com a direção de Newton encontrada, o algoritmo primal-dual itera por:

$$
w^{k+1}=w^{k}+\beta_{k} \Delta w^{k}
$$

ou seja,

$$
\begin{aligned}
& x^{k+1}=x^{k}+\beta_{k} \Delta x^{k} \\
& y^{k+1}=y^{k}+\beta_{k} \Delta y^{k}
\end{aligned}
$$




$$
\mathrm{z}^{\mathrm{k}+1}=\mathrm{z}^{\mathrm{k}}+\beta_{\mathrm{k}} \Delta \mathrm{z}^{\mathrm{k}}
$$

onde $\beta_{k}$ é o tamanho do passo a ser dado na direção $\Delta w^{k}$.

$\mathrm{Na}$ próxima seção, veremos como calcular o tamanho do passo $\beta$ e também como deverá se comportar o parâmetro de penalização $\mu$.

\subsection{Tamanho do Passo e o Parâmetro de Penalização}

Para que o ponto $\left(\mathrm{x}^{\mathrm{k}}, \mathrm{y}^{\mathrm{k}}, \mathrm{z}^{\mathrm{k}}\right)$ seja interior a cada iteração $\mathrm{k}$, o método primal-dual necessita de dois parâmetros $\sigma$ e $\tau$ tal que $0 \leq \tau<\sigma<1$, para controlar o parâmetro de penalização $\mu_{\mathrm{k}}$ e o tamanho do passo $\beta_{\mathrm{k}}$.

Para tanto, introduziremos as seguintes notações:

$$
\begin{aligned}
& \mathrm{f}_{\mathrm{i}}^{\mathrm{k}}=\mathrm{x}_{\mathrm{i}}^{\mathrm{k}} \mathrm{z}_{\mathrm{i}}^{\mathrm{k}} \quad(\mathrm{i}=1,2, \ldots, \mathrm{n}) \\
& \mathrm{f}_{\min }^{\mathrm{k}}=\min \left\{\mathrm{f}_{\mathrm{i}}^{\mathrm{k}} ; \mathrm{i}=1,2, \ldots, \mathrm{n}\right\} \\
& \left.\mathrm{f}_{\text {med }}^{\mathrm{k}}=\left\{\left(\sum_{\mathrm{i}=1}^{\mathrm{n}} \mathrm{f}_{\mathrm{i}}^{\mathrm{k}}\right) / \mathrm{n}\right\} \quad \quad \text { (a média das } \mathrm{f}_{\mathrm{i}}^{\mathrm{k} / \mathrm{s}}\right) \\
& \pi^{\mathrm{k}}=\mathrm{f}_{\text {med }}^{\mathrm{k}} / \mathrm{f}_{\text {min }}^{\mathrm{k}}
\end{aligned}
$$

onde $\pi^{\mathrm{k}}$ mede um desvio da curva $\Gamma$ a cada $\left(\mathrm{x}^{\mathrm{k}}, \mathrm{y}^{\mathrm{k}}, \mathrm{z}^{\mathrm{k}}\right) \in \stackrel{\circ}{\mathrm{S}} \times \stackrel{\circ}{\mathrm{T}}$.

Vemos também que $\pi^{\mathrm{k}} \geq 1$ e que $\left(\mathrm{x}^{\mathrm{k}}, \mathrm{y}^{\mathrm{k}}, \mathrm{z}^{\mathrm{k}}\right) \in \stackrel{\circ}{\mathrm{S}} \times \stackrel{\circ}{\mathrm{T}}$ também pertencerá à curva $\Gamma$ se, e somente se, $\pi^{\mathrm{k}}=1$.

Se fizermos uma escolha adequada dos parâmetros de controle, então a sequência de pontos $\left\{\left(\mathrm{x}^{\mathrm{k}}, \mathrm{y}^{\mathrm{k}}, \mathrm{z}^{\mathrm{k}}\right) \in \stackrel{\circ}{\mathrm{S}} \times \stackrel{\circ}{\mathrm{T}}\right\}$ gerada pelo algoritmo primal-dual satisfaz as seguintes inequações (ver [KOJ89]):

$$
\begin{aligned}
& c^{t} x^{k+1}-b^{t} y^{k+1} \leq\left(1-2 /\left(n-\pi^{k}\right)\right)\left(c^{t} x^{k}-b^{t} y^{k}\right) \\
& \pi^{k+1}-2 \leq(1-1 /(n+1))\left(\pi^{k}-2\right) \quad \text { se } \pi^{k}>2 \\
& \pi^{k+1} \leq 2 \text { se } \pi^{k} \leq 2
\end{aligned}
$$

A inequação (4.4.5) assegura que o gap de dualidade decresce monotonicamente. Das inequações (4.4.6) e (4.4.7) temos que o desvio $\pi^{\mathrm{k}}$ torna-se menor que $3 \mathrm{em}$ no máximo $\mathrm{O}\left(\mathrm{n} \log \pi^{0}\right)$ iterações, e que o gap de dualidade converge para zero linearmente com raio de convergência de no máximo $(1-2 /(3 n))$. Cada iteração requer $\mathrm{O}\left(\mathrm{n}^{3}\right)$ operações aritméticas.(ver [KOJ89]). 
Para reduzir o gap de dualidade, $\mathrm{nf}_{\text {med }}^{\mathrm{k}}$, escolhemos como parâmetro de penalização um número pequeno dado por:

$$
\mu^{\mathrm{k}}=\sigma \mathrm{f}_{\text {med }}^{\mathrm{k}}
$$

Isto implica, por (4.3.5), que $\mathrm{v}^{\mathrm{k}} \leq 0$.

Note das equações (4.3.4c) e (4.4.1) que,

$$
\mathrm{x}_{\mathrm{i}}^{\mathrm{k}} \Delta \mathrm{z}_{\mathrm{i}}^{\mathrm{k}}+\mathrm{z}_{\mathrm{i}}^{\mathrm{k}} \Delta \mathrm{x}_{\mathrm{i}}^{\mathrm{k}}=\mu^{\mathrm{k}}-\mathrm{f}_{\mathrm{i}}^{\mathrm{k}}
$$

As folgas complementares variam quadraticamente em termos do tamanho do passo $\beta$, e de (4.4.9) segue:

$$
\begin{array}{r}
f_{i}^{k}(\beta)=\left(x_{i}^{k}+\beta \Delta x_{i}^{k}\right)\left(z_{i}^{k}+\beta \Delta z_{i}^{k}\right)=f_{i}^{k}+\beta\left(\mu^{k}-f_{i}^{k}\right)+\beta^{2}\left(\Delta x_{i}^{k} \Delta z_{i}^{k}\right) \\
i=1,2, . ., n
\end{array}
$$

Além do mais, o gap de dualidade muda linearmente com $\beta$, isto é,

$$
\mathrm{f}_{\text {med }}^{\mathrm{k}}(\beta)=\left(\mathrm{x}^{\mathrm{k}}+\beta \Delta \mathrm{x}^{\mathrm{k}}\right)^{t}\left(\mathrm{z}^{\mathrm{k}}+\beta \Delta \mathrm{z}^{\mathrm{k}}\right) / \mathrm{n}=\mathrm{f}_{\text {med }}^{\mathrm{k}}+\beta\left(\mu^{\mathrm{k}}-\mathrm{f}_{\text {med }}^{\mathrm{k}}\right)
$$

A equação (4.4.11) é obtida levando em consideração que $\left(\Delta \mathrm{x}^{\mathrm{k}}\right)^{\mathrm{t}} \Delta \mathrm{z}^{\mathrm{k}}=0$, resultado da combinação de (4.3.4a) e (4.3.4b), mas para pontos factíveis, e da multiplicação destes por $\left(\Delta y^{k}\right)^{t}$ e $\left(\Delta x^{k}\right)^{t}$ respectivamente.

Ignorando o termo quadrático em (4.4.10) e reduzindo o valor $\mu^{\mathrm{k}}=\sigma \mathrm{f}_{\text {med }}^{\mathrm{k}}$ por um fator $\tau<\sigma$, podemos definir uma função linear,

$$
h^{k}(\beta)=f_{\min }^{k}+\beta\left(\tau f_{m e d}^{k}-f_{\text {min }}^{k}\right)
$$

Para controlar o parâmetro de desvio $\pi^{\mathrm{k}}$ enquanto reduzimos as folgas complementares, escolhemos:

$$
\begin{array}{r}
\alpha^{\mathrm{k}}=\max \left\{\bar{\beta} \mid \mathbf{f}_{\mathrm{i}}^{\mathrm{k}}(\beta) \geq \mathbf{h}^{\mathrm{k}}(\beta) \text { para todo } \beta \in(0, \bar{\beta}),\right. \\
0<\bar{\beta}<1, \quad \text { e } \mathrm{i}=1, \ldots, \mathrm{n}\}
\end{array}
$$

e o tamanho do passo $\beta^{\mathrm{k}}$ na k-ésima iteração é definida por: 


$$
\beta^{k}=\min \left\{1, \alpha^{k}\right\}
$$

A escolha de $\beta^{\mathrm{k}}$ depende da escolha de $0<\tau<1$ para assegurar a existência de $\alpha^{\mathrm{k}}$.

Desde que $\left(\mathrm{x}^{\mathrm{k}}, \mathrm{y}^{\mathrm{k}}, \mathrm{z}^{\mathrm{k}}\right) \in \stackrel{\circ}{\mathrm{S}} \times \stackrel{\circ}{\mathrm{T}}$ e $\left(\Delta \mathrm{x}^{\mathrm{k}}, \Delta \mathrm{y}^{\mathrm{k}}, \Delta \mathrm{z}^{\mathrm{k}}\right)$ seja a solução de (4.3.4), com $\mathrm{t}^{\mathrm{k}}=0$ e $\mathrm{u}^{\mathrm{k}}=0$, sabemos que $A \mathrm{x}^{\mathrm{k}+1}=\mathrm{b}$ e $\mathrm{A}^{\mathrm{t}} \mathrm{y}^{\mathrm{k}+1}+\mathrm{z}^{\mathrm{k}+1}=\mathrm{c}$. Além disso, a definição de $\alpha^{\mathrm{k}}$ em (4.4.13) implica que $\mathrm{x}^{\mathrm{k}+1}>0$ e $\mathrm{z}^{\mathrm{k}+1}>0$. Ou seja, $\left(\mathrm{x}^{\mathrm{k}+1}, \mathrm{y}^{\mathrm{k}+1}, \mathrm{z}^{\mathrm{k}+1}\right) \in \stackrel{\circ}{\mathrm{S}} \times \stackrel{\circ}{\mathrm{T}}$.

Após as discussões anteriores, introduziremos na próxima seção, o algoritmo básico do método primal-dual.

\subsection{Algoritmo Primal-Dual}

\section{FASE 1}

Passo 1 (Inicialização): Seja $k=0$. Encontre uma solução inicial $\left(\mathrm{x}^{0}, \mathrm{y}^{0}, \mathrm{z}^{0}\right) \in \stackrel{\circ}{\mathrm{S}} \times \stackrel{\circ}{\mathrm{T}}$ (veja seção 4.6). Sejam $\varepsilon>0$ uma tolerância para o gap de dualidade e $\sigma$ e $\tau$ os parâmetros de controle tal que $0 \leq \tau<\sigma<1$.

FASE 2

Passo 2 (Verificação da otimalidade): Se $c^{t} x^{k}-b^{t} y^{k}<\varepsilon$, então PARE.

Passo 3 (Determinação da direção de busca): Defina $f_{i}^{k}=x_{i}^{k} z_{i}^{k}$

$$
\begin{aligned}
& \mathrm{f}_{\text {med }}^{\mathrm{k}}=\left\{\left(\sum_{\mathrm{i}=1}^{\mathrm{n}} \mathrm{f}_{\mathrm{i}}^{\mathrm{k}}\right) / \mathrm{n}\right\} \\
& \mathrm{f}_{\min }^{\mathrm{k}}=\min \left\{\mathrm{f}_{\mathrm{i}}^{\mathrm{k}} ; \mathrm{i}=1,2, \ldots, \mathrm{n}\right\} \\
& \mu^{\mathrm{k}}=\sigma \mathrm{f}_{\text {med }}^{\mathrm{k}}
\end{aligned}
$$

Resolva o sistema linear:

$$
\begin{aligned}
& {\left[\begin{array}{cc}
-X_{k}^{-1} Z_{k} & A^{t} \\
A & 0
\end{array}\right]\left[\begin{array}{c}
\Delta x^{k} \\
\Delta y^{k}
\end{array}\right]=\left[\begin{array}{c}
c-A^{t} y^{k}-\mu^{k} X_{k}^{-1} e \\
b-A x^{k}
\end{array}\right]} \\
& \Delta z^{k}=\mu^{k} X_{k}^{-1} e-z^{k}-X_{k}^{-1} Z_{k} \Delta x^{k}
\end{aligned}
$$

Passo 4 (Cálculo do tamanho do passo): Calcule:

$$
\begin{aligned}
& \mathrm{h}^{\mathrm{k}}(\beta)=\mathrm{f}_{\text {min }}^{\mathrm{k}}+\beta\left(\tau \mathrm{f}_{\text {med }}^{\mathrm{k}}-\mathrm{f}_{\text {min }}^{\mathrm{k}}\right) \\
& \alpha^{\mathrm{k}}=\max \left\{\bar{\beta} \mid \mathrm{f}_{\mathrm{i}}^{\mathrm{k}}(\beta) \geq \mathrm{h}^{\mathrm{k}}(\beta) \text { para todo } \beta \in(0, \bar{\beta}), 0<\bar{\beta}<1, \text { e } \mathrm{i}=1, \ldots \mathrm{n}\right\} \\
& \beta^{\mathrm{k}}=\min \left\{1, \alpha^{\mathrm{k}}\right\}
\end{aligned}
$$

Passo 5 (Nova solução): Sejam:

$$
\begin{aligned}
& \mathrm{x}^{\mathrm{k}+1}=\mathrm{x}^{\mathrm{k}}+\beta_{\mathrm{k}} \Delta \mathrm{x}^{\mathrm{k}} \\
& \mathrm{y}^{\mathrm{k}+1}=\mathrm{y}^{\mathrm{k}}+\beta_{\mathrm{k}} \Delta \mathrm{y}^{\mathrm{k}} \\
& \mathrm{z}^{\mathrm{k}+1}=\mathrm{z}^{\mathrm{k}}+\beta_{\mathrm{k}} \Delta \mathrm{z}^{\mathrm{k}}
\end{aligned}
$$

Faça $\mathrm{k} \leftarrow \mathrm{k}+1$ e repita o PASSO 2. 


\subsection{Solução Inicial (Fase I)}

Nesta seção apresentamos um programa primal e dual artificiais para inicialização do algoritmo primal-dual [KOJ89].

Escolhemos um ponto arbitrário $\left(\mathrm{x}^{0}, \mathrm{y}^{0}, \mathrm{z}^{0}\right) \in \mathfrak{R}^{\mathrm{n \times m \times n}}$ tal que $\mathrm{x}^{0}>0$ e $\mathrm{z}^{0}>0$. Se $\mathrm{Ax}^{0}=\mathrm{be} \mathrm{A}^{\mathrm{t}} \mathrm{y}^{0}+\mathrm{z}^{0}=\mathrm{c}$ então esse ponto é factível e será solução inicial do algoritmo primaldual. Caso contrário, considere o seguinte par de problemas lineares primal e dual artificiais:

$$
\begin{aligned}
&\left(\mathbf{P}_{\mathbf{A}}\right) \text { Minimize } \mathbf{c}^{\mathrm{t} x}+\psi \mathbf{x}_{\mathrm{n}+1} \\
& \text { sujeito à }\left\{\begin{array}{c}
\mathbf{A x}+\left(\mathbf{b}-\mathbf{A} \mathbf{x}^{0}\right) \mathbf{x}_{\mathrm{n}+1}=\mathbf{b} \\
\left(\mathbf{A}^{\mathrm{t}} \mathbf{y}^{0}+\mathbf{z}^{0}-\mathbf{c}\right)^{\mathrm{t}} \mathbf{x}+\mathbf{x}_{\mathrm{n}+2}=\xi \\
\left(\mathbf{x}, \mathbf{x}_{\mathrm{n}+1}, \mathbf{x}_{\mathrm{n}+2}\right) \geq \mathbf{0}
\end{array}\right.
\end{aligned}
$$

onde $x_{n+1}$ e $x_{n+2}$ são variáveis reais artificiais e $\psi$ e $\xi$ são números positivos suficientemente grandes.

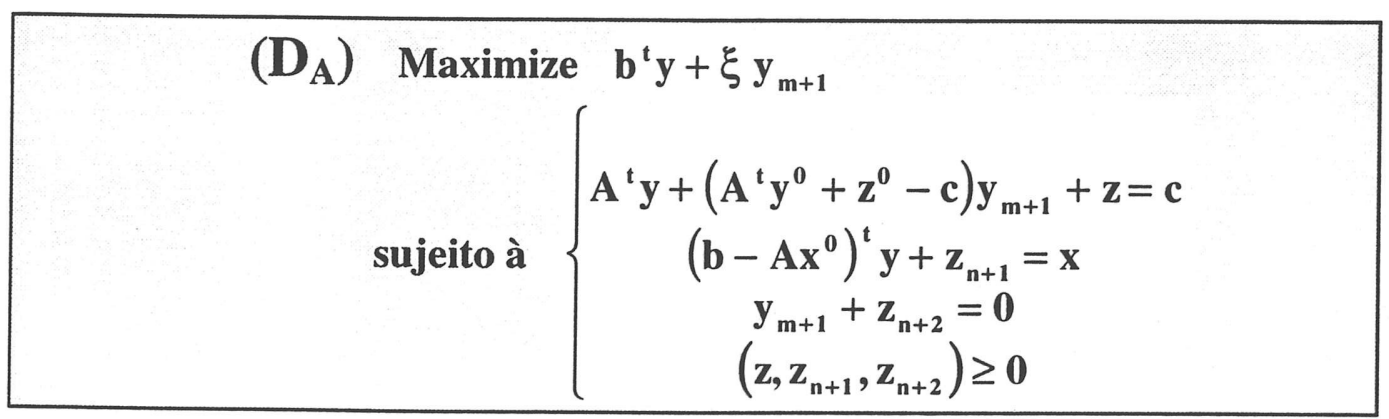

onde $\mathrm{y}_{\mathrm{m}+1}, \mathrm{z}_{\mathrm{n}+1}$ e $\mathrm{z}_{\mathrm{n}+2}$ são variáveis reais artificiais.

Precisamos tomar $\psi$ e $\xi$ satisfazendo

$$
\begin{aligned}
& \xi>\left(A^{t} y^{0}+z^{0}-c\right)^{t} x^{0} \\
& \psi>\left(b-A x^{0}\right)^{t} y^{0}
\end{aligned}
$$

de modo que $\left(\mathrm{x}^{0}, \mathrm{x}_{\mathrm{n}+1}^{0}, \mathrm{x}_{\mathrm{n}+2}^{0}\right)$ e $\left(\mathrm{y}^{0}, \mathrm{y}_{\mathrm{m}+1}^{0}, \mathrm{z}^{0}, \mathrm{z}_{\mathrm{n}+1}^{0}, \mathrm{z}_{\mathrm{n}+2}^{0}\right)$ são soluções factívieis de $\left(\mathrm{P}_{\mathrm{A}}\right)$ e $\left(\mathrm{D}_{\mathrm{A}}\right)$, respectivamente, onde

$$
\begin{aligned}
& x_{n+1}^{0}=1 \\
& x_{n+2}^{0}=\xi-\left(A^{t} y^{0}+z^{0}-c\right)^{t} x^{0} \\
& y_{m+1}=-1
\end{aligned}
$$




$$
\begin{aligned}
& \mathrm{z}_{\mathrm{n}+1}^{0}=\psi-\left(\mathrm{b}-\mathrm{Ax^{0 }}\right)^{\mathrm{t}} \mathrm{y}^{0} \\
& \mathrm{z}_{\mathrm{n}+2}^{0}=1
\end{aligned}
$$

Portanto, podemos aplicar o algoritmo para os problemas $\left(\mathrm{P}_{\mathrm{A}}\right)$ e $\left(\mathrm{D}_{\mathrm{A}}\right)$ com essas soluções iniciais.

Esta versão do algoritmo primal-dual gera uma sequência de pontos estritamente positivos que segue para a solução ótima, embora não permaneça na curva $\mathrm{S} \times \mathrm{T}$. É importante colocar que, até o momento, não há uma prova de convergência rigorosa dessa versão, mas tem sido bastante empregado em pacotes comerciais [FAN93].

Na próximo capítulo será feito uma extensão do algoritmo primal-dual, visto neste capítulo, para problemas de programação linear por partes. 


\section{CAPítulo 5}

\section{EXTENSÃO DO MÉTODO PRIMAL-DUAL}

\subsection{Introdução}

Nos últimos anos vários pesquisadores têm feito modificações no algoritmo básico primal-dual para melhorar seu desempenho assim como muitos procuraram estendê-lo a outros tipos de problemas. Neste capítulo fazemos uma extensão do Método Primal-Dual para um problema linear por partes com variáveis canalizadas, tomando por base o trabalho de R. Fourer [FOU91]. Seguiremos procedimentos análogos aos utilizados nos demais capítulos.

\subsection{Definição de um problema linear por partes}

Considere o seguinte problema linear por partes:

\begin{tabular}{|c|c|}
\hline $\begin{array}{r}\left(\mathbf{P}_{\text {LPC }}\right) \text { Minimize } \\
\text { sujeito à }\end{array}$ & $\begin{array}{l}\mathbf{c}^{\mathbf{t}} \mathbf{x}+\mathbf{v}^{ \pm} \mathbf{s} \\
\left\{\begin{array}{c}\mathbf{A x}+\mathbf{s}=\mathbf{b} \\
\mathbf{u}^{-} \leq \mathbf{x} \leq \mathbf{u}^{+}\end{array}\right.\end{array}$ \\
\hline
\end{tabular}

onde $\mathrm{v}^{ \pm} \mathrm{S}=\sum_{\mathrm{i}=1}^{\mathrm{m}} \mathrm{v}_{\mathrm{i}}^{ \pm} \mathrm{S}_{\mathrm{i}}$ é uma soma de funções lineares por partes convexas com: 


$$
v_{i}^{ \pm} s_{i}= \begin{cases}v_{i}^{+} s_{i} & \text { se } s_{i} \geq 0 \\ v_{i}^{-} s_{i} & \text { se } s_{i} \leq 0\end{cases}
$$

$e v_{i}^{-}<v_{i}^{+}$. A figura 5.2.1 a seguir ilustra a função convexa $v_{i}^{ \pm} s_{i}$.

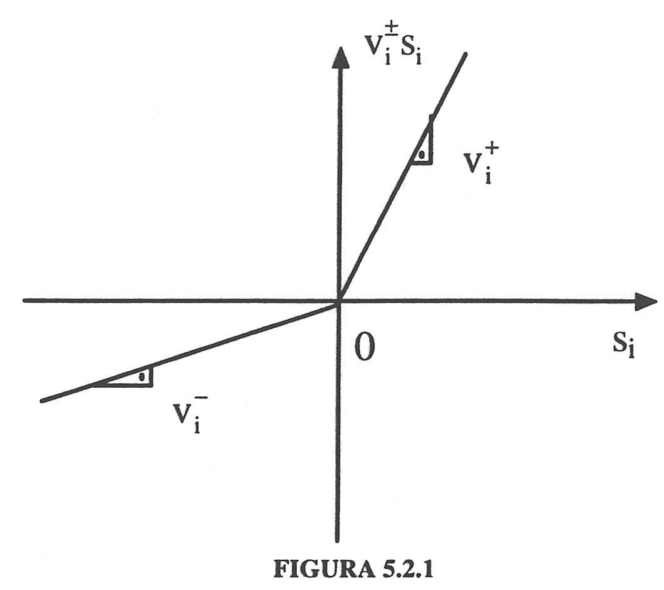

Esta estrutura é bem abrangente em relação as formas de apresentação, bastando no caso de uma restrição de $(\geq)$ tomar $v_{i}^{-}=0$ e $v_{i}^{+} \rightarrow \infty$ e no caso de uma restrição de $(=)$ tomar $\mathrm{v}_{\mathrm{i}}^{-} \rightarrow-\infty \mathrm{e} \mathrm{v}_{\mathrm{i}}^{+} \rightarrow \infty$.

\subsection{O problema dual e condições de otimalidade}

Já vimos no capítulo 2 o procedimento para se encontrar o problema dual de um problema com variáveis canalizadas. Agora, procederemos analogamente para encontrar o problema dual do problema linear por partes com variáveis canalizadas $\left(\mathrm{P}_{\mathrm{LPC}}\right)$.

Considere a função lagrangeana do problema $\left(\mathrm{P}_{\mathrm{LPC}}\right)$ definida abaixo:

$$
L(x, s, y)=\left(c^{t} x+v^{ \pm} s\right)+y^{t}(b-A x-s)
$$

Logo, a função dual é:

$$
l(y)=\min _{u^{-} \leq x \leq u^{+}}\left[c^{t} x+v^{ \pm} s+y^{t}(b-A x-s)\right]
$$

A função dual, por conseguinte a solução do problema em (5.3.2), pode ser expressa pelo desmembramento da função lagrangeana componente a componente, e usando o fato das variáveis $\mathrm{x}_{\mathrm{j}}$ serem desacopladas, temos: 


$$
\begin{aligned}
1(y)= & \min _{u^{-} \leq x \leq u^{+}}\left[\left(c^{t}-y^{t} A\right) x+v^{ \pm} s-y^{t} s+y^{t} b\right]= \\
& =\min _{u^{-} \leq x \leq u^{+}}\left[\left(c^{t}-y^{t} A\right) x\right]+\min _{s}\left[v^{ \pm} s-y^{t} s\right]+y^{t} b= \\
& =\min _{u_{j}^{-} \leq x_{j} \leq u_{j}^{+}} \sum_{j=1}^{n}\left(c_{j}-y^{t} a_{j}\right) x_{j}+\min _{s_{i}} \sum_{i=1}^{m}\left(v_{i}^{ \pm} s_{i}-y_{i} s_{i}\right)+y^{t} b= \\
& =\sum_{j=1}^{n} \min _{u_{j}^{-} \leq x_{j} \leq u_{j}^{+}}\left(c_{j}-y^{t} a_{j}\right) x_{j}+\sum_{i=1}^{m} \min _{s_{i}}\left(v_{i}^{ \pm} s_{i}-y_{i} s_{i}\right)+y^{t} b
\end{aligned}
$$

onde $a_{j}, j=1, \ldots, n$ são as colunas da matriz A.

Considere o sub-problema em (5.3.3):

$$
\min _{u_{j}^{-} \leq x_{j} \leq u_{j}^{+}}\left(c_{j}-y^{t} a_{j}\right) x_{j}
$$

Para simplificar a notação, seja:

$$
z^{t}=c^{t}-y^{t} A
$$

Desta forma, o sub-problema a ser analisado é:

$$
\min _{u_{j}^{-} \leq x_{j} \leq u_{j}^{+}} z_{j} x_{j}
$$

Se $z_{j}>0$ então $\min _{u_{j}^{-} \leq x_{i} \leq u_{j}^{+}} z_{j} x_{j}=z_{j} u_{j}{ }^{-}$

Se $z_{j}=0$ então $\min _{u_{j}^{-} \leq x_{j} \leq u_{j}^{+}} z_{j} x_{j}=0$ para $\forall x_{j} \in\left[u_{j}{ }^{-}, u_{j}{ }^{+}\right]$

Se $\mathrm{z}_{\mathrm{j}}<0$ então $\min _{\mathrm{u}_{\mathrm{i}}^{-} \leq \mathrm{x}_{\mathrm{i}} \leq \mathrm{u}_{\mathrm{j}}^{+}} \mathrm{z}_{\mathrm{j}} \mathrm{x}_{\mathrm{j}}=\mathrm{z}_{\mathrm{j}} \mathrm{u}_{\mathrm{j}}^{+}$

Tomamos agora o outro sub-problema em (5.3.3):

$$
\min _{s_{i}} v_{i}^{ \pm} s_{i}-y_{i} s_{i}
$$

Se $s_{i} \geq 0$ então $\min _{s_{i} \geq 0}\left[v_{i}^{ \pm} s_{i}-y_{i} s_{i}\right]=\min _{s_{i} \geq 0}\left(v_{i}^{+}-y_{i}\right) s_{i}$, por definição. Entretanto, para que exista um mínimo, é necessário que $\left(\mathrm{v}_{\mathrm{i}}^{+}-\mathrm{y}_{\mathrm{i}}\right) \geq 0$.

Se $s_{i} \leq 0$ então $\min _{s_{i} \leq 0}\left[v_{i}^{ \pm} s_{i}-y_{i} s_{i}\right]=\min _{s_{i} \leq 0}\left(v_{i}^{-}-y_{i}\right) s_{i}$ por definição. Para que exista um mínimo, é necessário que $\left(\mathrm{v}_{\mathrm{i}}^{-}-\mathrm{y}_{\mathrm{i}}\right) \leq 0$.

Desta forma, para garantir que o mínimo exista, devemos levar em consideração as seguintes desigualdades: $\left(\mathrm{v}_{\mathrm{i}}^{+}-\mathrm{y}_{\mathrm{i}}\right) \geq 0$ e $\left(\mathrm{v}_{\mathrm{i}}^{-}-\mathrm{y}_{\mathrm{i}}\right) \leq 0$, ou seja, 


$$
\mathrm{v}_{\mathrm{i}}^{-} \leq \mathrm{y}_{\mathrm{i}} \leq \mathrm{v}_{\mathrm{i}}^{+}
$$

e

$$
\min _{s_{i}} v_{i}^{ \pm} s_{i}-y_{i} s_{i}=0
$$

Das expressões (5.3.3), (5.3.7)-(5.3.9) e (5.3.12), obtemos a função dual e observando as condições (5.3.5) e (5.3.11), obtemos o problema dual de $\left(\mathrm{P}_{\mathrm{LPC}}\right)$, dado a seguir:

$$
\begin{aligned}
& \text { Maximize } 1(y)=u^{\mp} z+y^{t} b \\
& \text { sujeito à }\left\{\begin{array}{c}
z^{t}=c^{t}-y^{t} A \\
v^{-} \leq y \leq v^{+}
\end{array}\right.
\end{aligned}
$$

ou seja,

$$
\begin{gathered}
\text { (D LPC) } \\
\text { sujeito à }\left\{\begin{array}{l}
\mathbf{A}^{\mathrm{t}} \mathrm{y}+\mathrm{z}=\mathrm{c} \\
\mathbf{v}^{-} \leq \mathrm{y} \leq \mathbf{v}^{+}
\end{array}\right.
\end{gathered}
$$

onde $\mathrm{u}^{\mp} \mathrm{z}=\sum_{\mathrm{j}=1}^{\mathrm{n}} \mathrm{u}_{\mathrm{j}}^{\mp} \mathrm{z}_{\mathrm{j}}$ é uma soma de funções lineares por partes côncavas com:

$$
u_{j}^{\mp} z_{j}= \begin{cases}u_{j}^{-} z_{j} & \text { se } z_{j} \geq 0 \\ u_{j}^{+} z_{j} & \text { se } z_{j} \leq 0\end{cases}
$$

onde $\mathrm{u}^{-}<\mathrm{u}^{+}$são os limitantes da variável primal x. A figura 5.3.1 a seguir ilustra a função $\mathrm{u}_{\mathrm{j}}^{\mp} \mathrm{z}_{\mathrm{j}}$.

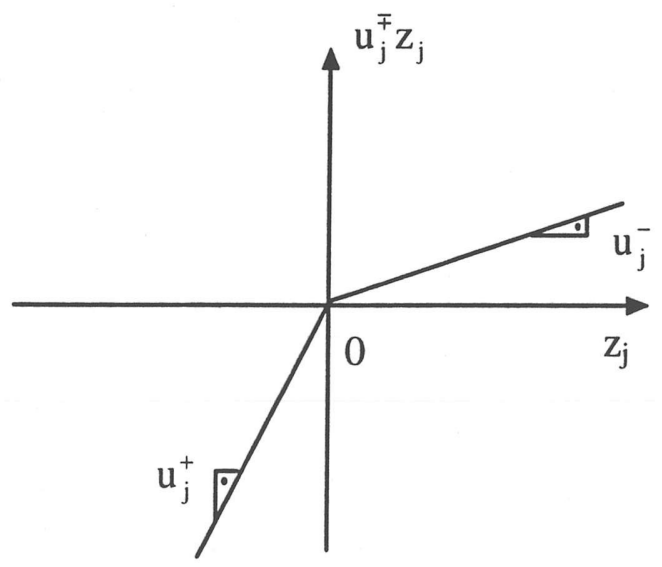

FIGURA 5.3.1 
A seguir, expressaremos as condições de otimalidade de acordo com o teorema 2.2.1. Para tanto, escreveremos os problemas $\left(\mathrm{P}_{\mathrm{LPC}}\right)$ e $\left(\mathrm{D}_{\mathrm{LPC}}\right)$ com uma redefinição de variáveis, em formato linear.

Sejam:

$$
\begin{aligned}
& s=s^{+}-s^{-}, \quad s^{+} \geq 0, \quad s^{-} \geq 0 \\
& z=z^{-}-z^{+}, \quad z^{-} \geq 0, \quad z^{+} \geq 0
\end{aligned}
$$

A inversão de sinais na decomposição de z segue, por conveniência, a definição da função dual.

Desta forma, os formatos linearizados dos problemas $\left(\mathrm{P}_{\mathrm{LPC}}\right)$ e $\left(\mathrm{D}_{\mathrm{LPC}}\right)$ são

$$
\begin{aligned}
& \left(\mathrm{P}_{\mathrm{LPC} 2}\right) \quad \text { Minimize } \mathrm{c}^{\mathrm{t}} \mathrm{x}+\mathrm{v}^{+} \mathrm{s}^{+}-\mathrm{v}^{-} \mathrm{s}^{-} \\
& \text {sujeito à }\left\{\begin{array}{c}
A x+s^{+}-s^{-}=b \\
-x \geq-u^{+} \\
x \geq u^{-} \\
s^{+} \geq 0 \\
s^{-} \geq 0
\end{array}\right. \\
& \left(D_{\text {LPC2 }}\right) \text { Maximize } b^{t} y+u^{-} z^{-}-u^{+} z^{+} \\
& \text {sujeito à }\left\{\begin{array}{c}
\mathrm{A}^{\mathrm{t}} \mathrm{y}+\mathrm{z}^{-}-\mathrm{z}^{+}=\mathrm{c} \\
\mathrm{y} \leq \mathrm{v}^{+} \\
-\mathrm{y} \leq-\mathrm{v}^{-} \\
-\mathrm{z}^{-} \leq 0 \\
-\mathrm{z}^{+} \leq 0
\end{array}\right.
\end{aligned}
$$

A equivalência entre os problemas deveriam requerer $s_{i}^{+} s_{i}^{-}=0$ e $z_{i}^{+} z_{i}^{-}=0$. Entretanto, estas condições podem ser abandonadas pois as colunas de $s_{i}^{+}$e $s_{i}^{-}$(respectivamente $z_{j}^{+}$e $z_{j}^{-}$) são linearmente dependentes e na otimalidade deverão ser verificadas.

Desenvolveremos o método primal-dual para os problemas canalizados no formato linearizado, de forma que os conceitos e procedimentos vistos no capítulo 4 serão válidos neste capítulo. Os procedimentos serão análogos.

Para expressar as condições KKT para os problemas $\left(\mathrm{P}_{\mathrm{LPC} 2}\right)$ e $\left(\mathrm{D}_{\mathrm{LPC} 2}\right)$, definiremos

$$
\begin{array}{lc}
\mathrm{X}_{-}=\operatorname{diag}\left(\mathrm{x}_{\mathrm{j}}-\mathrm{u}_{\mathrm{j}}^{-}\right) & \mathrm{X}_{+}=\operatorname{diag}\left(\mathrm{u}_{\mathrm{j}}^{+}-\mathrm{x}_{\mathrm{j}}\right) \\
\mathrm{Y}_{-}=\operatorname{diag}\left(\mathrm{y}_{\mathrm{i}}-\mathrm{v}_{\mathrm{i}}^{-}\right) & \mathrm{Y}_{+}=\operatorname{diag}\left(\mathrm{v}_{\mathrm{i}}^{+}-\mathrm{y}_{\mathrm{i}}\right)
\end{array}
$$


A seguir, a factibilidade primal, a factibilidade dual e as folgas complementares das condições KKT para que os vetores $(\mathrm{x}, \mathrm{s}) \operatorname{com} \mathrm{u}^{-} \leq \mathrm{x} \leq \mathrm{u}^{+} \mathrm{e}(\mathrm{y}, \mathrm{z})$ com $\mathrm{v}^{-} \leq \mathrm{y} \leq \mathrm{v}^{+}$sejam ótimos.

$$
\begin{aligned}
& A x+s^{+}-s^{-}=b \\
& A^{t} y+z^{-}-z^{+}=c \\
& Z^{-} X_{-} e=0 \\
& Z^{+} X_{+} e=0 \\
& S^{-} Y_{-} e=0 \\
& S^{+} Y_{+} e=0
\end{aligned}
$$

com:

$$
\begin{array}{lll}
\mathrm{S}^{+}=\operatorname{diag}\left(\mathrm{s}^{+}\right) & \text {e } & \mathrm{S}^{-}=\operatorname{diag}\left(\mathrm{s}^{-}\right) \\
\mathrm{Z}^{-}=\operatorname{diag}\left(\mathrm{z}^{-}\right) & \text {e } & \mathrm{Z}^{+}=\operatorname{diag}\left(\mathrm{z}^{+}\right)
\end{array}
$$

Para $\mathrm{u}^{-}<\mathrm{x}<\mathrm{u}^{+}, \mathrm{s}^{+}>0 \mathrm{e} \mathrm{s}^{-}>0$ de $\left(\mathrm{P}_{\mathrm{LPC} 2}\right)$ aplicamos a técnica da função barreira logarítmica e obtemos:

\begin{tabular}{|c|}
\hline$\left(\mathbf{P}_{\mu 2}\right)$ Minimize $\mathbf{c}^{\mathrm{t}} \mathbf{x}+\mathrm{v}^{+} \mathbf{s}^{+}-\mathbf{v}^{-} \mathbf{s}^{-}-\mu \mathrm{p}\left(\mathrm{x}, \mathrm{s}^{+}, \mathbf{s}^{-}\right)$ \\
sujeito à $\left\{\begin{array}{c}\mathbf{A x}+\mathbf{s}^{+}-\mathbf{s}^{-}=\mathbf{b} \\
-\mathbf{x}>-\mathbf{u}^{+} \\
\mathbf{x}>\mathbf{u}^{-} \\
\mathbf{s}^{+}>\mathbf{0} \\
\mathbf{s}^{-}>\mathbf{0}\end{array}\right.$
\end{tabular}

onde $\mu>0$ é um parâmetro de barreira e,

$$
\mathrm{p}\left(\mathrm{x}, \mathrm{s}^{+}, \mathrm{s}^{-}\right)=\sum_{\mathrm{i}=1}^{\mathrm{n}} \ln \left(\mathrm{x}_{\mathrm{i}}-\mathrm{u}_{\mathrm{i}}^{-}\right)+\sum_{\mathrm{i}=1}^{\mathrm{n}} \ln \left(\mathrm{u}_{\mathrm{i}}^{+}-\mathrm{x}_{\mathrm{i}}\right)+\sum_{\mathrm{i}=1}^{\mathrm{m}} \ln \mathrm{s}_{\mathrm{i}}^{+}+\sum_{\mathrm{i}=1}^{\mathrm{m}} \ln \mathrm{s}_{\mathrm{i}}^{-}
$$

As condições KKT para o problema não linear $\left(\mathrm{P}_{\mu 2}\right)$ são dados por:

$$
\begin{aligned}
& A x+s^{+}-s^{-}=b \\
& A^{t} y+z^{-}-z^{+}=c \\
& Z^{-} X_{-} e=\mu e
\end{aligned}
$$




$$
\begin{aligned}
& \mathbf{Z}^{+} \mathbf{X}_{+} \mathbf{e}=\mu \mathrm{e} \\
& \mathbf{S}^{-} \mathbf{Y}_{-} \mathbf{e}=\mu \mathrm{e} \\
& \mathbf{S}^{+} \mathbf{Y}_{+} \mathbf{e}=\mu \mathrm{e}
\end{aligned}
$$

Aplicando o método de Newton no sistema (5.3.18) encontramos a direção do método primal-dual, que será apresentado na seção seguinte.

\subsection{Direção}

Seja

$$
F(w)=\left[\begin{array}{c}
A x+s^{+}-s^{-}-b \\
A^{t} y+z^{-}-z^{+}-c \\
Z^{-} X_{-} e-\mu e \\
Z^{+} X_{+} e-\mu e \\
S^{-} Y_{-} e-\mu e \\
S^{+} Y_{+} e-\mu e
\end{array}\right] \text { onde } w=\left(x, s^{+}, s^{-}, y, z^{-}, z^{+}\right)
$$

O sistema (5.3.18) é, desta forma, $F(w)=0$.

Pelo método de Newton fazemos uma aproximação linear para $\mathrm{F}$ usando expansão de série de Taylor no ponto $\mathrm{w}^{\mathrm{k}}$, obtendo:

$$
F\left(w^{k}\right)+\nabla F\left(w^{k}\right) \Delta w^{k}=0
$$

por onde encontramos a direção de Newton $\Delta w^{k}$.

Analogamente à seção 4.3., o método de Newton aplicado ao sistema $F(w)=0$ implica em:

$$
\left[\begin{array}{c}
\mathrm{Ax}^{\mathrm{k}}+\mathrm{s}_{\mathrm{k}}^{+}-\mathrm{s}_{\mathrm{k}}^{-}-\mathrm{b} \\
\mathrm{A}^{\mathrm{t}} \mathrm{y}^{\mathrm{k}}+\mathrm{z}_{\mathrm{k}}^{-}-\mathrm{z}_{\mathrm{k}}^{+}-\mathrm{c} \\
\mathrm{Z}_{\mathrm{k}}^{-} \mathrm{X}_{-}^{\mathrm{k}} \mathrm{e}-\mu \mathrm{e} \\
\mathrm{Z}_{\mathrm{k}}^{+} \mathrm{X}_{+}^{\mathrm{k}} \mathrm{e}-\mu \mathrm{e} \\
\mathrm{S}_{\mathrm{k}}^{-} \mathrm{Y}_{-}^{\mathrm{k}} \mathrm{e}-\mu \mathrm{e} \\
\mathrm{S}_{\mathrm{k}}^{+} \mathrm{X}_{+}^{\mathrm{k}} \mathrm{e}-\mu \mathrm{e}
\end{array}\right]+\left[\begin{array}{cccccc}
\mathrm{A} & \mathrm{I} & -\mathrm{I} & 0 & 0 & 0 \\
0 & 0 & 0 & \mathrm{~A}^{\mathrm{t}} & \mathrm{I} & -\mathrm{I} \\
\mathrm{Z}_{\mathrm{k}}^{-} & 0 & 0 & 0 & \mathrm{X}_{-}^{\mathrm{k}} & 0 \\
-\mathrm{Z}_{\mathrm{k}}^{+} & 0 & 0 & 0 & 0 & \mathrm{X}_{+}^{\mathrm{k}} \\
0 & 0 & \mathrm{Y}_{-}^{\mathrm{k}} & \mathrm{S}_{\mathrm{k}}^{-} & 0 & 0 \\
0 & \mathrm{Y}_{+}^{\mathrm{k}} & 0 & -\mathrm{S}_{\mathrm{k}}^{+} & 0 & 0
\end{array}\right]\left[\begin{array}{c}
\Delta \mathrm{x}^{\mathrm{k}} \\
\Delta \mathrm{s}_{\mathrm{k}}^{+} \\
\Delta \mathrm{s}_{\mathrm{k}}^{-} \\
\Delta \mathrm{y}^{\mathrm{k}} \\
\Delta \mathrm{z}_{\mathrm{k}}^{-} \\
\Delta \mathrm{z}_{\mathrm{k}}^{+}
\end{array}\right]=\left[\begin{array}{l}
0 \\
0 \\
0 \\
0 \\
0 \\
0
\end{array}\right]
$$


Com algumas substituições é possível escrever o sistema acima em termos apenas de $\Delta \mathrm{x}^{\mathrm{k}}$ e $\Delta \mathrm{y}^{\mathrm{k}}$. Logo, o sistema (5.4.1) se escreve como:

$$
\left[\begin{array}{cc}
-\mathbf{Z}_{\mathbf{k}}^{-}\left(\mathbf{X}_{-}^{\mathbf{k}}\right)^{-1}+\mathbf{Z}_{\mathbf{k}}^{+}\left(\mathbf{X}_{+}^{\mathbf{k}}\right)^{-1} & \mathbf{A}^{\mathrm{t}} \\
\mathbf{A} & \mathbf{S}_{\mathbf{k}}^{+}\left(\mathbf{Y}_{+}^{\mathbf{k}}\right)^{-1}+\mathbf{S}_{\mathbf{k}}^{-}\left(\mathbf{Y}_{-}^{\mathbf{k}}\right)^{-1}
\end{array}\right]\left[\begin{array}{l}
\Delta \mathbf{x} \\
\Delta \mathbf{y}
\end{array}\right]=\left[\begin{array}{c}
\mu\left[\left(\mathbf{X}_{+}^{\mathbf{k}}\right)^{-1}-\left(\mathbf{X}_{-}^{\mathbf{k}}\right)^{-1}\right] \mathbf{e}+\left(\mathbf{c}-\mathbf{A}^{\mathbf{t}} \mathbf{y}\right) \\
\mu\left[\left(\mathbf{Y}_{-}^{\mathbf{k}}\right)^{-1}-\left(\mathbf{Y}_{+}^{\mathbf{k}}\right)^{-1}\right] \mathbf{e}+(\mathbf{b}-\mathbf{A x})
\end{array}\right]
$$

Portanto a solução do sistema (5.4.2) nos fornece a direção $\left(\Delta \mathrm{x}^{\mathrm{k}}, \Delta \mathrm{y}^{\mathrm{k}}\right)$ do método primal-dual para o problema linear por partes canalizado. Observe que mesmo tendo um aumento no número de variáveis quando da linearização do problema canalizado, o sistema linear a ser resolvido para encontrar a direção de busca permanece com a mesma estrutura do algoritmo primal-dual, do capítulo 4 , devido às simplificações.

\subsection{Considerações adicionais}

$\mathrm{Na}$ seção anterior, determinamos a direção $(\Delta x, \Delta y)$, e consequentemente, $\left(\Delta \mathrm{s}^{+}, \Delta \mathrm{s}^{-}\right) \mathrm{e}\left(\Delta \mathrm{z}^{-}, \Delta \mathrm{z}^{+}\right)$, que serão usadas para computar uma nova iteração $\mathrm{k}$. Esta nova iteração é definida por

$$
\begin{aligned}
& \mathrm{x}^{\mathrm{k}}=\mathrm{x}^{\mathrm{k}-1}+\alpha_{\mathrm{p}} \Delta \mathrm{x}^{\mathrm{k}-1} \\
& \mathrm{~s}_{\mathrm{k}}^{-}=\mathrm{s}_{\mathrm{k}-1}^{-}+\alpha_{\mathrm{p}} \Delta \mathrm{s}_{\mathrm{k}-1}^{-} \\
& \mathrm{s}_{\mathrm{k}}^{+}=\mathrm{s}_{\mathrm{k}-1}^{+}+\alpha_{\mathrm{p}} \Delta \mathrm{s}_{\mathrm{k}-1}^{+} \\
& \mathrm{y}^{\mathrm{k}}=\mathrm{y}^{\mathrm{k}-1}+\alpha_{\mathrm{d}} \Delta \mathrm{y}^{\mathrm{k}-1} \\
& \mathrm{z}_{\mathrm{k}}^{-}=\mathrm{z}_{\mathrm{k}-1}^{-}+\alpha_{\mathrm{d}} \Delta \mathrm{z}_{\mathrm{k}-1}^{-} \\
& \mathrm{z}_{\mathrm{k}}^{+}=\mathrm{z}_{\mathrm{k}-1}^{+}+\alpha_{\mathrm{d}} \Delta \mathrm{z}_{\mathrm{k}-1}^{+}
\end{aligned}
$$

Segundo R. Fourer [FOU91], as soluções obtidas nas iterações tendem a satisfazer a factibilidade primal e dual das condições de otimalidade dadas por

$$
\begin{aligned}
& A x^{k}+s_{k}^{+}-s_{k}^{-}=b \\
& A^{t} y^{k}+z_{k}^{-}-z_{k}^{+}=c
\end{aligned}
$$

e o valor ótimo pode ser limitado (veja capítulo 2, seção 2) por

$$
\mathbf{b}^{\mathrm{t}} \mathbf{y}^{\mathbf{k}}+\mathbf{u}^{-} \mathbf{z}_{\mathrm{k}}^{-}-\mathbf{u}^{+} \mathbf{z}_{\mathrm{k}}^{+} \leq \text {valor ótimo } \leq \mathbf{c}^{\mathrm{t}} \mathbf{x}^{\mathrm{k}}+\mathrm{v}^{+} \mathbf{s}_{\mathrm{k}}^{+}-\mathbf{v}^{-} \mathbf{s}_{\mathrm{k}}^{-}
$$


$\mathrm{O}$ retorno às variáveis originais $\mathrm{s}=\mathrm{s}^{+}-\mathrm{s}^{-} \mathrm{e} \mathrm{z}=\mathrm{z}^{-}-\mathrm{z}^{+}$da formulação linear por partes produz limitantes mais justos. Nestes termos, a nova iteração é dada por

$$
\begin{aligned}
& \mathrm{s}_{\mathrm{k}}=\mathrm{s}_{\mathrm{k}}^{+}-\mathrm{s}_{\mathrm{k}}^{-}=\mathrm{s}_{\mathrm{k}-1}+\alpha_{\mathrm{p}}\left(\Delta \mathrm{s}_{\mathrm{k}-1}^{+}-\Delta \mathrm{s}_{\mathrm{k}-1}^{-}\right) \\
& \mathrm{z}_{\mathrm{k}}=\mathrm{z}_{\mathrm{k}}^{-}-\mathrm{z}_{\mathrm{k}}^{+}=\mathrm{z}_{\mathrm{k}-1}+\alpha_{\mathrm{d}}\left(\Delta \mathrm{z}_{\mathrm{k}-1}^{-}-\Delta \mathrm{z}_{\mathrm{k}-1}^{+}\right)
\end{aligned}
$$

onde $\mathrm{s}_{\mathrm{k}}^{+}, \mathrm{s}_{\mathrm{k}}^{-}, \mathrm{z}_{\mathrm{k}}^{-}, \mathrm{z}_{\mathrm{k}}^{+}$são estritamente positivos.

Os limitantes nas variáveis originais são dados por

$$
\mathbf{b}^{t} \mathbf{y}^{k}+\mathbf{u}^{\mp} \mathbf{z}_{k} \leq \text { valor ótimo } \leq \mathbf{c}^{t} \mathbf{x}^{k}+\mathbf{v}^{ \pm} \mathbf{S}_{k}
$$

Verificaremos que esses limitantes são mais justos mostrando, inicialmente, que $\mathrm{u}^{\mp} \mathrm{z}_{\mathrm{k}}>\mathrm{u}^{-} \mathrm{z}_{\mathrm{k}}^{-}-\mathrm{u}^{+} \mathrm{z}_{\mathrm{k}}^{+}$e, em seguida, que $\mathrm{v}^{ \pm} \mathrm{s}_{\mathrm{k}}<\mathrm{v}^{+} \mathrm{s}_{\mathrm{k}}^{+}-\mathrm{v}^{-} \mathrm{z}_{\mathrm{k}}^{-}$.

Por (5.5.7) e pela definição da função, temos:

$$
u^{\mp} z_{k}=\sum_{j} u_{j}^{\mp}\left(z_{k}\right)
$$

onde $u_{j}^{\mp}\left(z_{k}\right)_{j}= \begin{cases}u_{j}^{-}\left[\left(z_{k}\right)_{j}^{-}-\left(z_{k}\right)_{j}^{+}\right] & \text {se }\left(z_{k}\right)_{j}^{-}-\left(z_{k}\right)_{j}^{+}>0 \\ u_{j}^{+}\left[\left(z_{k}\right)_{j}^{-}-\left(z_{k}\right)_{j}^{+}\right] & \text {se }\left(z_{k}\right)_{j}^{-}-\left(z_{k}\right)_{j}^{+}<0\end{cases}$

a) $\operatorname{Se}\left(\mathrm{z}_{\mathrm{k}}\right)_{\mathrm{j}}^{-}-\left(\mathrm{z}_{\mathrm{k}}\right)_{\mathrm{j}}^{+}>0$ então $\mathrm{u}_{\mathrm{j}}^{\mp}\left(\mathrm{z}_{\mathrm{k}}\right)_{\mathrm{j}}=\mathrm{u}_{\mathrm{j}}^{-}\left[\left(\mathrm{z}_{\mathrm{k}}\right)_{\mathrm{j}}^{-}-\left(\mathrm{z}_{\mathrm{k}}\right)_{\mathrm{j}}^{+}\right]=$

$$
=u_{j}^{-}\left(z_{k}\right)_{j}^{-}-u_{j}^{+}\left(z_{k}\right)_{j}^{+}+\left(u_{j}^{+}-u_{j}^{-}\right)\left(z_{k}\right)_{j}^{+}
$$

Como $u_{j}^{+}-u_{j}^{-}>0 e\left(z_{k}\right)_{j}^{+}>0$, segue que $u_{j}^{\mp}\left(z_{k}\right)_{j}>u_{j}^{-}\left(z_{k}\right)_{j}^{-}-u_{j}^{+}\left(z_{k}\right)_{j}^{+}$.

b) $\operatorname{Se}\left(\mathrm{z}_{\mathrm{k}}\right)_{\mathrm{j}}^{-}-\left(\mathrm{z}_{\mathrm{k}}\right)_{\mathrm{j}}^{+}<0$ então $\mathrm{u}_{\mathrm{j}}^{\mp}\left(\mathrm{z}_{\mathrm{k}}\right)_{\mathrm{j}}=\mathrm{u}_{\mathrm{j}}^{+}\left[\left(\mathrm{z}_{\mathrm{k}}\right)_{\mathrm{j}}^{-}-\left(\mathrm{z}_{\mathrm{k}}\right)_{\mathrm{j}}^{+}\right]=$

$$
=\left(u_{j}^{+}-u_{j}^{-}\right)\left(z_{k}\right)_{j}^{-}+u_{j}^{-}\left(z_{k}\right)_{j}^{-}-u_{j}^{+}\left(z_{k}\right)_{j}^{+}
$$

Como $u_{j}^{+}-u_{j}^{-}>0 e\left(z_{k}\right)_{j}^{-}>0$, segue que $u_{j}^{\mp}\left(z_{k}\right)_{j}>u_{j}^{-}\left(z_{k}\right)_{j}^{-}-u_{j}^{+}\left(z_{k}\right)_{j}^{+}$.

Por $\boldsymbol{a}$ ) e $\boldsymbol{b}$ ) obtemos $\mathrm{u}^{\mp} \mathrm{z}_{\mathrm{k}}>\mathrm{u}^{-} \mathrm{z}_{\mathrm{k}}^{-}-\mathrm{u}^{+} \mathrm{z}_{\mathrm{k}}^{+}$. 
A outra desigualdade decorre da definição da função em questão e de (5.5.6), de onde temos

$$
\mathrm{v}^{ \pm} \mathrm{s}_{\mathrm{k}}=\sum_{\mathrm{i}} \mathrm{v}_{\mathrm{i}}^{ \pm}\left[\left(\mathrm{s}_{\mathrm{k}}\right)_{\mathrm{i}}^{+}-\left(\mathrm{s}_{\mathrm{k}}\right)_{\mathrm{i}}^{-}\right]
$$

onde $v_{i}^{ \pm}\left[\left(s_{k}\right)_{i}^{+}-\left(s_{k}\right)_{i}^{-}\right]=\left\{\begin{array}{cc}v_{i}^{+}\left[\left(s_{k}\right)_{i}^{+}-\left(s_{k}\right)_{i}^{-}\right] & s e\left(s_{k}\right)_{i}^{+}>\left(s_{k}\right)_{i}^{-} \\ v_{i}^{-}\left[\left(s_{k}\right)_{i}^{+}-\left(s_{k}\right)_{i}^{-}\right] & s e\left(s_{k}\right)_{i}^{+}<\left(s_{k}\right)_{i}^{-}\end{array}\right.$

Por uma análise semelhante observada em $\boldsymbol{a}$ ) e $\boldsymbol{b}$ ) obtemos $\mathrm{v}^{ \pm} \mathrm{s}_{\mathrm{k}}<\mathrm{v}^{+} \mathrm{s}_{\mathrm{k}}^{+}-\mathrm{v}^{-} \mathrm{s}_{\mathrm{k}}^{-}$.

Portanto

$$
\mathbf{b}^{\mathrm{t}} \mathbf{y}^{\mathrm{k}}+\mathbf{u}^{-} \mathbf{z}_{\mathrm{k}}^{-}-\mathbf{u}^{+} \mathbf{z}_{\mathrm{k}}^{+}<\mathbf{b}^{\mathrm{t}} \mathbf{y}^{\mathrm{k}}+\mathbf{u}^{\mp} \mathbf{z}_{\mathrm{k}} \leq \text { valor ótimo } \leq \mathbf{c}^{\mathrm{t}} \mathbf{x}^{\mathrm{k}}+\mathbf{v}^{ \pm} \mathbf{s}_{\mathrm{k}}<\mathbf{c}^{\mathrm{t}} \mathbf{x}^{\mathrm{k}}+\mathbf{v}^{+} \mathbf{s}_{\mathrm{k}}^{+}-\mathbf{v}^{-} \mathbf{s}_{\mathrm{k}}^{-}
$$

As desigualdades entre as funções lineares por partes e linearizadas tornam-se mais significativas quando a solução está longe da fronteira.

Com as considerações adicionais encerramos este capítulo, ficando para o último capítulo (Capítulo 6) as conclusões, as considerações finais do trabalho, bem como as perspectivas em face da bibliografia consultada. 


\section{Capítulo 6}

\section{CONCLUSÕES E CONSIDERAÇÕES FINAIS}

Inicialmente devemos ressaltar que este trabalho não teve a pretensão de estudar todos os tipos de problemas de programação linear de pontos interiores.

Foi feito uma leitura geral da bibliografia encontrada a respeito dos métodos de pontos interiores e dentre eles selecionamos alguns. A ênfase foi dada aos métodos de pontos interiores primais-duais, entretanto isto não significa a caracterização da superioridade desse método. A atenção foi dada a esse algoritmo devido a frequência com que vários pesquisadores publicaram trabalhos sobre esse tema, direta ou indiretamente.

O método primal-dual e outros métodos de pontos interiores ainda estão em fase de pesquisas e experimentações. Mostrou-se eficiente em alguns casos como em alguns problemas de grande porte, entretanto em outros casos, o método simplex foi melhor. Implementações práticas e experimentos numéricos desenvolvidos por K.A. McShane, C.L. Monna e D.F. Shanno e por I.J. Lustig, R.E. Marsten e D.F. Shanno, por exemplo, demostraram que algoritmos primais-duais podem ser computacionalmente bem eficientes (ver [TAP95]), mas por enquanto a tendência é a complementaridade entre os dois tipos de métodos: o simplex e o de pontos interiores.

Outra discussão é em relação a classificação dos métodos de pontos interiores devido a várias interpretações e variações. Um exemplo disso são as denominações dadas ao método primal-dual, ora chamado de método path-following, ora método escala-afim primal-dual, ou mesmo somente de método primal-dual. Dado este fato, para fazer este trabalho e dar noções de alguns dos métodos mais pesquisados, escolhemos seguir a classificação dada por S. Fang e S. Puthenpura [FAN93] e apresentamos os algoritmos básicos de: 
-Escala-afim primal
-Escala-afim dual
-Escala-afim primal-dual

Este último, por conveniência, foi chamado somente de método primal-dual.

Uma importante subárea da programação matemática, ignorada por tempos, parece estar sendo resgatada por alguns pesquisadores que estão evidenciando suas inúmeras aplicações tanto na área teórica como em situações reais [MAR96]. Sendo assim, no Capítulo 5, estudamos uma extensão do algoritmo primal-dual a problemas lineares por partes baseado no trabalho de R. Fourer [FOU91].

Devido às várias interpretações de métodos de pontos interiores, alguns pesquisadores procuraram unificar os vários trabalhos. Exemplos desse fato são os trabalhos de D.F. Shanno e A. Bagchi [SHA91] e mais recentemente o trabalho de R.J. Vanderbei, A. Duarte e B. Yang [VAN94].

Shanno e Bagchi mostram que os métodos projetivo, afim, centros ou path-following, são todos variantes do método de barreira logarítmica aplicados aos problemas primal, dual e primal-dual. Mostram também que todos esses métodos de pontos interiores solucionam um sistema de equações do tipo:

$$
\left(\mathrm{AD}^{2} \mathrm{~A}^{\mathrm{t}}\right) \Delta=\xi
$$

onde $\mathrm{D}$ é matriz diagonal e $\xi$ uma combinação linear de vetores de centralização e gradiente escalado.

Vanderbei, Duarte e Yang fizeram uma comparação numérica e algorítmica entre alguns métodos de pontos interiores, e dentre eles o método primal-dual.

Como observação, colocamos que o método primal-dual estudado nesta dissertação entra na categoria de método path-following no trabalho de Vanderbei, Duarte e Yang. E mais, que a classificação de método de escala-afim primal-dual é dado ao problema derivado do método path-following tomando $\mu=0$ como parâmetro de barreira.

Somente como uma citação, mencionamos que a convergência da sequência de iteração dos algoritmos de pontos interiores primal-dual foi estudada por alguns pesquisadores como R.A. Tapia, Y. Zhang e Y.Ye [TAP95], os quais mostram a convergência quadrática sob certas condições.

Outros campos têm sido investigados em relação ao método primal-dual. Pesquisadores como M. Kojima, N. Megiddo e S. Mizuno [KOJ93] e S. Mizuno [MIZ94], que publicaram trabalhos sobre algoritmos de pontos interiores infactíveis primais-duais e sua complexidade polinomial, outros que fizeram extensões a problemas quadráticos convexos[MON89-2], 
problemas convexos, problemas de complementaridade não-linear e linear (estes últimos citados em [KOJ93]), não deixam dúvidas que este tipo de método ainda reserva surpresas, bem como a área de métodos de pontos interiores, cujo desenvolvimento e aprimoramento tende a se estender por mais alguns anos até se estabilizar. 


\section{Referências Bibliográficas}

[ADL89] Adler, I., Karmarkar, N., Resende, M. G. C., Veiga, G., An implementation of Karmarkar's algorithm for linear programming, Mathematical Programming 44, 297 335, 1989. Errata: Mathematical Programming 50, p.415, 1991.

[ARE90] Arenales, M. N., Balbo, A. R., Um método de pontos interiores com mudança de escala para a programação linear com restrições canalizadas, São Carlos, 1990, Notas do ICMSC-USP 66, Instituto de Ciências Matemáticas de São Carlos, Universidade de São Paulo.

[BAL91] Balbo, A. R., Métodos de pontos interiores com mudança de escala para a programação linear, São Carlos, 1991, 79 p., Dissertação(Mestrado), Instituto de Ciências Matemáticas de São Carlos, Universidade de São Paulo.

[BAZ77] Bazaraa, M. S., Jarvis, J. J., Linear Programming and Network Flows, John Wiley \& Sons, USA, 1977.

[CHV83] Chvátal, V., Linear Programming,W. H. Freeman and Company, New York, 1983.

[FAN93] Fang, S., Puthenpura, S., Linear Optimization and Extensions: Theory and Algorithms, Prentice Hall, New Jersey, 1993.

[FOU91] Fourer, R., Notes on Alternative Standard Forms for Primal-Dual Path-Following Interior-Point Methods in Linear Programming, Working Paper, Departament of Industrial Engineering and Management Sciences, Northwestern University, Evanston, Chicago, USA, 1991. 
[FRI55] Frisch, K. R., The logarithmic potential method of convex programming, Memorandum, University Institute of Economics, Oslo, Norway, 1955.

[GON89] Gonzaga, C. C., Algoritmos de Pontos Interiores em Programação Linear, 69 p., Texto de Acompanhamento, I EBO - Escola Brasileira de Otimização, 1989.

[GON90] Gonzaga, C. C., Polynomial affine algorithms for linear programming, Mathematical Programming 49, 7-21, 1990.

[GON92] Gonzaga, C. C., Path-following methods for linear programming, Siam Review 34, n.2, 167-224, 1992.

[KAR84] Karmarkar, N., A new polynomial-time algorithm for linear programming, Combinatorica 4, 373-395, 1984.

[KOJ89] Kojima, M., Mizuno, S., Yoshise, A., A primal-dual interior point algorithm for linear programming, In: N. Megiddo, ed., Progress in Mathematical Programming, InteriorPoint and Related Methods, Springer-Verlag, New York, 29-47, 1989.

[KOJ93] Kojima, M., Megiddo, N., Mizuno, S., A primal-dual infeasible-interior-point algorithm for linear programming, Mathematical Programming 61, 263-280, 1993.

[LUE84] Luenberger, D. G., Linear and Nonlinear Programming, $2^{0}$. Ed., Addison-Wesley Publishing Company, USA, 1984.

[MAR96] Marins, F. A. S., Perin Filho, C., Programação Linear por Partes: revisão teórica e aplicações, In: VIII CLAIO - Latin-Iberian-American Congress on Operations Research and System Engineering/ XXVIII SBPO - Simpósio Brasileiro de Pesquisa Operacional, Rio de Janeiro, Anais, v.2, 498-503, 1996.

[MAR91] Martinhon, C. A. J., Análise da Velocidade de Convergência dos Métodos Primais de Redução de Pontencial em Programação Linear, Rio de Janeiro, 1991, 104 p., Dissertação(Mestrado), COPPE, Universidade Federal do Rio de Janeiro.

[MIZ94] Mizuno, S., Polynomiality of infeasible-interior-point algorithms for linear programming, Mathematical Programming 67, 109-119, 1994. 
